\title{
First-order electroweak phase transition in a complex singlet model with $\mathbb{Z}_{3}$ symmetry
}

\author{
Cheng-Wei Chiang ${ }^{a, b}$ and Bo-Qiang Lu ${ }^{a}$ \\ ${ }^{a}$ Department of Physics, National Taiwan University, \\ Taipei, Taiwan 10617, Republic of China \\ ${ }^{b}$ Institute of Physics, Academia Sinica, \\ Taipei, Taiwan 11529, Republic of China \\ E-mail: chengwei@phys.ntu.edu.tw, bqlu@phys.ntu.edu.tw
}

Abstract: We consider an extension of the Standard Model with a complex singlet scalar, where a global $U(1)$ symmetry is explicitly broken to $\mathbb{Z}_{3}$ symmetry. We study the two-step electroweak phase transition in the model and find that it can be of first-order if the heavy scalar mass falls in the range of $1-2 \mathrm{TeV}$ and the mixing angle $|\theta| \gtrsim 0.2\left(11.5^{\circ}\right)$. The Higgs signal strength measurements at the LHC, on the other hand, restrict the mixing angle $|\theta| \lesssim 0.4\left(23^{\circ}\right)$. Future colliders including high-luminosity LHC can probe the remaining parameter space of first-order phase transition in this scenario. After the $\mathrm{U}(1)$ symmetry breaking, the pseudo-Goldstone boson becomes a dark matter candidate due to a hidden $\mathbb{Z}_{2}$ symmetry of the model. We find that the pseudo-Goldstone boson can make up a small fraction of the observed dark matter and escape from the constraints of current direct detection. We also show that the stochastic gravitational wave signals from the phase transition are potentially discoverable with future space-based interferometers.

Keywords: Phase transitions, Beyond Standard Model, Dark matter, Higgs physics, Hadron-Hadron scattering (experiments)

ARXIV EPRINT: 1912.12634 


\section{Contents}

1 Introduction $\quad 2$

2 The model with $\mathbb{Z}_{3}$ symmetry 3

2.1 Field-dependent masses 4

2.2 Stationary points 5

2.2.1 Stationary points along $h$-axis 5

2.2.2 Stationary points along $s$-axis 6

2.2.3 Stationary points at $(h \neq 0, s \neq 0) \quad 6$

2.3 Parameters 7

3 Effective potential $\quad 7$

4 Parameter space for electroweak phase transition $\quad 8$

4.1 Two-step phase transition $\quad 9$

$\begin{array}{lll}4.2 & \text { Searching scheme } & 10\end{array}$

$\begin{array}{lll}4.3 & \text { Parameter distributions } & 11\end{array}$

5 Vacuum stability and perturbativity 16

6 Experimental constraints $\quad \mathbf{1 8}$

$\begin{array}{lll}6.1 & \text { Electroweak precision observables } & 18\end{array}$

$\begin{array}{lll}6.2 & \text { Higgs signal strengths } & 20\end{array}$

6.3 Results 22

7 Dark matter phenomenology 23

7.1 Dark matter relic density 23

$\begin{array}{ll}7.2 \text { Direct detection } & 25\end{array}$

8 Gravitational waves $\quad 26$

$\begin{array}{ll}8.1 & \text { Gravitational wave parameters }\end{array}$

$\begin{array}{ll}8.2 \text { Gravitational wave spectrum } & 28\end{array}$

8.3 Space-based interferometers 30

9 Gauge dependence $\quad 31$

10 Summary and conclusion $\quad 35$

A Field-dependent mass matrix at finite temperature $\quad 36$

$\begin{array}{ll}\text { B Renormalization group equations } & 37\end{array}$

$\begin{array}{ll}\text { C Counter-terms } & 38\end{array}$

$\begin{array}{ll}\text { D Decay width } & 38\end{array}$

E Sensitivity of space-based interferometers $\quad 39$ 


\section{Introduction}

The Standard Model (SM) of particle physics has been completed since the discovery of the Higgs boson at the LHC in 2012 [1, 2]. However, it is widely believed that new physics is required to explain various phenomena beyond SM, such as the existence of dark matter $(\mathrm{DM})$ and the origin of neutrino mass and mixing. We concern ourselves in this work two prominent particle physics puzzles. One is the origin of baryon number asymmetry in the Universe [3]. In the content of electroweak (EW) baryogenesis [4-7], the baryon number asymmetry is generated outside the EW broken phase and then captured by the bubbles' expansion in the progress of electroweak phase transition (EWPT). Furthermore, a sufficiently strong first-order EWPT is required to suppress the washout of baryon number asymmetry through sphalerons $[7,8]$. However, the EWPT in the SM with the observed Higgs boson mass is found to be a crossover [9]. The other is the existence of DM. There is overwhelming evidence from cosmological and astrophysical observations that show about $85 \%$ of the matter in the Universe is DM, instead of the normal matter made from SM particles $[3,10-12]$. The most popular class of DM candidates is that of weakly interacting massive particles (WIMPs), which lies in the mass range of $1-1000 \mathrm{GeV}[13,14]$. These particles decouple from the thermal bath as the early Universe expands and cools and finally reach the appropriate relic density as observed now.

One of the simplest models to trigger a strong first-order EWPT is to introduce a new bosonic degree of freedom to the scalar potential of SM. Various Higgs portal models with (or without) a DM candidate have been widely studied [15-47]. The extension of SM with a complex singlet scalar for the above-mentioned purpose was proposed and studied in refs. $[15,16]$, followed by extensive researches on its implications in subsequent works [16-19]. In the Higgs-portal model with a real singlet scalar as the DM candidate, the scalar is stabilized by a $\mathbb{Z}_{2}$ symmetry and cannot have a vacuum expectation value (VEV) [48]. DM phenomena related to this model have been studied in refs. [20-27]. A previous study [49] also shows that such a model cannot trigger a sufficiently strong firstorder EWPT except when a large number of singlet scalars $(\sim 10)$ are introduced. In the model with a global $\mathrm{U}(1)$ group explicitly broken down to $\mathbb{Z}_{2}$ symmetry, ref. [50] shows that all phase transitions leading to the correct vacuum are of the second order. Furthermore, ref. [51] shows that a large portion of the parameter space for the $\mathbb{Z}_{2}$ symmetry model will be ruled out by the required bubble nucleation condition, $S\left(T_{n}\right) / T_{n} \sim 140$.

By now, no obvious evidence for WIMPs has been observed in both DM direct detections and collider searches. In particular, the recent constraints from LUX [52], PandaXII [53], and XENON1T [54] tell us that the DM interaction cross section with protons and neutrons is extraordinarily tiny, less than about $10^{-45}-10^{-46} \mathrm{~cm}^{2}$, low enough to rule out most of WIMP DM models. Refs. [16-19, 55-59] show that there is a novel mechanism for suppressing the direct detection cross section if the global U(1) symmetry of Higgs portal model is softly broken to $\mathbb{Z}_{2}$ symmetry by a mass term. Such a suppression is due to a cancellation in the DM-nucleon scattering amplitude between the SM-like and new Higgs boson contributions at zero momentum transfer. Although the cancellation is spoiled at loop level, the scattering contribution from one loop is trivial and the conclusion remains practically unchanged $[60,61]$. 
In this work we are concerned with one of the simplest extensions of SM with a complex singlet scalar. In the model, the $\mathrm{U}(1)$ symmetry is softly broken by a cubic term to $\mathbb{Z}_{3}$ symmetry (see also recent works $[46,47]$ ). The real part of the complex scalar acquires a VEV, while the imaginary component (pseudo-Goldstone boson) plays the role of DM candidate due to a residual $\mathbb{Z}_{2}$ symmetry in the scalar potential. As shown in a previous study [48], such a cubic term could generally induce a potential barrier at tree level and trigger a strong first-order EWPT. We find that in the two-step EWPT scenario and for the heavy scalar mass falling in the range of $1-2 \mathrm{TeV}$, the model with a Higgs mixing angle satisfying $|\theta| \gtrsim 0.2\left(11.5^{\circ}\right)$ can induce a strong first-order EWPT and the stochastic gravitational wave $(\mathrm{GW})$ signals produced from the phase transition are potentially discoverable by future space-based interferometers, such as LISA [62], DECIGO [63] and BBO [64].

This work is presented as follows. In sections 2 and 3, we describe our model and study detailed properties of the model at tree level. In section 4, we present our search scheme and results for the strength of the EWPT from a comprehensive scan of the parameter space. We study the bounds of vacuum stability and perturbativity in the parameter space in section 5. We take into account the constraints from electroweak precision observables and Higgs searches at the colliders in section 6. The DM phenomenology of the pseudo-Goldstone boson $\chi$ is studied in section 7. The discussions of GW production along with the first-order cosmological phase transition and its detection by future space-based interferometers are given in section 8. In section 9, we study the effects of gauge dependence in our results and conclusions. We summarize our findings in section 10. Some detailed formulas and parameters are collected in the appendices: appendix A gives the field-dependent mass matrices of the particles at finite temperature; appendix B provides the renormalizable group equations of the parameters in the scalar potential, and shows the dependence of the critical temperature and the corresponding vacuum expectation value on the renormalization scale; appendix $\mathrm{C}$ provides the coefficients of counter-terms in the scalar potential; appendix D gives the partial decay widths of the SM-like Higgs and heavy scalar; and appendix E discusses the sensitivities of space-based interferometers.

\section{The model with $\mathbb{Z}_{3}$ symmetry}

We consider an extension of the SM with just a complex gauge-singlet scalar field $S$ which transforms under a global $\mathbb{Z}_{3}$ transformation as $S \rightarrow \exp (i 2 \pi / 3) S$. Imposing the $\mathbb{Z}_{3}$ symmmetry associated with $S$, we can write down the most general renormalizable scalar potential with softly $\mathrm{U}(1)$ symmetry breaking:

$$
V(H, S)=-\mu_{h}^{2}|H|^{2}+\lambda_{h}|H|^{4}+\frac{1}{2} \lambda_{m}|H|^{2}|S|^{2}+\frac{1}{2} \mu_{s}^{2}|S|^{2}+\frac{1}{6} \mu_{3}\left(S^{3}+S^{* 3}\right)+\frac{1}{4} \lambda_{s}|S|^{4},
$$

where $H$ denotes the SM Higgs doublet and $\mu_{3}$ is assumed to be real. The symmetry of global U(1) transformation $S \rightarrow \exp (i \vartheta) S$ ( $\vartheta$ is an arbitrary phase) is softly broken by the $\mu_{3}$ term to the $\mathbb{Z}_{3}$ symmetry, i.e., the potential remains unchanged only under those transformations with a rotation angle $\vartheta=2 n \pi / 3$, where $n$ is an integer. Notice that the Hermiticity of the potential implies a symmetry under the transformation $S \rightarrow S^{*}$, which 
turns into a $\mathbb{Z}_{2}$ symmetry for the imaginary component $\chi$ of $S(\chi \rightarrow-\chi)$. This ensures the stability of $\chi$ and makes it a DM candidate. It is worth mentioning that the usual $\mathbb{Z}_{2}$ symmetry models do not prohibit a term proportional to $|H|^{2} S^{2}$, which could also softly break the U(1) symmetry but at the same time spoil the cancellation mechanism [57]. Such a term is not allowed by the $\mathbb{Z}_{3}$ symmetry in our model, which shows an advantage over the $\mathbb{Z}_{2}$ symmetry models. Furthermore, this tree-level potential boasts analytical solutions which may explicitly reveal some of properties of the model.

We note in passing that the breakdown of a discrete symmetry during EWPT in the early universe can potentially lead to the problematic EW-scale cosmic domain walls [65] whose gravitational effects may result in unacceptable anisotropy in the cosmic microwave background (CMB) radiation [66]. Depending on the stability and evolution of such domain walls, several mechanisms have been proposed to avoid these quandaries [66-72]. One of the mechanisms proposed in refs. [66-69] was to assume that the discrete symmetry was not exact but approximate. One can introduce a so-called "bias" term to the scalar potential to explicitly break the discrete symmetry. This term lifts the degenerate vacua and induces a difference in the energy density between these vacua. This difference in the energy density has effects on the wall as a volume pressure and finally leads to the decay of the wall when the pressure becomes comparable to the surface tension of the wall [73]. In a recent paper [74], the authors took our model and applied the approximate symmetry mechanism to solve the domain wall problem. It was shown that there were two peaks in the GW spectrum, one from the first-order EWPT and the other from the domain wall decay.

\section{$2.1 \quad$ Field-dependent masses}

The Higgs and singlet scalar can be expanded around their classical backgrounds as

$$
H=\left(\begin{array}{c}
G^{+} \\
\frac{1}{\sqrt{2}}\left(h+i G^{0}\right)
\end{array}\right), \quad S=s+i \chi,
$$

where $G^{ \pm}, G^{0}$, and $\chi$ are the Goldstone bosons after spontaneous symmetry breaking. At zero temperature, the VEVs for the two scalars are $\left.v \equiv\langle H\rangle\right|_{T=0}$ and $\left.w \equiv\langle S\rangle\right|_{T=0}$, respectively. We immediately obtain the tree-level potential in terms of the fields $h$ and $s$

$$
V(h, s)=-\frac{1}{2} \mu_{h}^{2} h^{2}+\frac{1}{4} \lambda_{h} h^{4}+\frac{1}{4} \lambda_{m} h^{2} s^{2}+\frac{1}{2} \mu_{s}^{2} s^{2}+\frac{1}{3} \mu_{3} s^{3}+\frac{1}{4} \lambda_{s} s^{4} .
$$

Then the field-dependent mass matrix of the scalar bosons is given by

$$
\mathcal{M}^{2}(h, s)=\left(\begin{array}{ll}
\mathcal{M}_{h h}^{2} & \mathcal{M}_{h s}^{2} \\
\mathcal{M}_{s h}^{2} & \mathcal{M}_{s s}^{2}
\end{array}\right), \text { with }\left\{\begin{array}{l}
\mathcal{M}_{h h}^{2}=-\mu_{h}^{2}+3 \lambda_{h} h^{2}+\frac{1}{2} \lambda_{m} s^{2} \\
\mathcal{M}_{s s}^{2}=\mu_{s}^{2}+3 \lambda_{s} s^{2}+2 \mu_{3} s+\frac{1}{2} \lambda_{m} h^{2} \\
\mathcal{M}_{h s}^{2}=\mathcal{M}_{s h}^{2}(h, s)=\lambda_{m} h s
\end{array}\right.
$$

The tree-level potential can now be rewritten as

$$
V(h, s)=\frac{1}{2} \Phi^{\dagger} \mathcal{M}^{2}(h, s) \Phi,
$$


where $\Phi^{\dagger}=(h, s)$. With an orthogonal rotation

$$
\left(\begin{array}{l}
\mathcal{H} \\
\mathcal{S}
\end{array}\right)=\left(\begin{array}{cc}
\cos \theta & -\sin \theta \\
\sin \theta & \cos \theta
\end{array}\right)\left(\begin{array}{l}
h \\
s
\end{array}\right)
$$

from the $(h, s)^{T}$ basis to the physical basis $(\mathcal{H}, \mathcal{S})$, one finds the physical masses given by

$$
\begin{aligned}
& M_{\mathcal{H}}^{2}(h, s)=\frac{1}{2}\left(\mathcal{M}_{h h}^{2}+\mathcal{M}_{s s}^{2}-\sqrt{\left(\mathcal{M}_{h h}^{2}-\mathcal{M}_{s s}^{2}\right)^{2}+4 \mathcal{M}_{h s}^{2} \mathcal{M}_{s h}^{2}}\right), \\
& M_{\mathcal{S}}^{2}(h, s)=\frac{1}{2}\left(\mathcal{M}_{h h}^{2}+\mathcal{M}_{s s}^{2}+\sqrt{\left(\mathcal{M}_{h h}^{2}-\mathcal{M}_{s s}^{2}\right)^{2}+4 \mathcal{M}_{h s}^{2} \mathcal{M}_{s h}^{2}}\right) .
\end{aligned}
$$

The field-dependent mass of pseudo-Goldstone boson $\chi$ is

$$
M_{\chi}^{2}=\mu_{s}^{2}+\lambda_{s} s^{2}+\frac{1}{2} \lambda_{m} h^{2}-2 \mu_{3} s .
$$

Other field-dependent masses of the SM particles are given in appendix A.

\subsection{Stationary points}

We now search for the local minima of the tree-level scalar potential. An interesting scenario is when the potential has two local minima: the EW symmetry broken one located at $(v, w)$ and the EW symmetric one located at $\left(0, w_{0}\right)$. The tadpole conditions of the potential are

$$
\begin{aligned}
& \frac{\partial V}{\partial h}=0 \Rightarrow\left\{h=0, \quad \text { or } h^{2}=\frac{1}{2 \lambda_{h}}\left(2 \mu_{h}^{2}-\lambda_{m} s^{2}\right)\right\}, \\
& \frac{\partial V}{\partial s}=0 \Rightarrow\left\{s=0, \quad \text { or } \quad h^{2}=-\frac{2}{\lambda_{m}}\left(\mu_{s}^{2}+\mu_{3} s+\lambda_{s} s^{2}\right)\right\} .
\end{aligned}
$$

Besides, vacuum stability demands the following bounds on parameters in the potential $[75,76]$

$$
\lambda_{h}>0, \quad \lambda_{s}>0, \quad \lambda_{h} \lambda_{s}>\frac{1}{4} \lambda_{m}^{2} .
$$

\subsubsection{Stationary points along $h$-axis}

The stationary point along the $h$-axis is given by

$$
s=0, \quad h_{ \pm}= \pm \sqrt{\frac{\mu_{h}^{2}}{\lambda_{h}}} .
$$

The condition for a physical vacuum, i.e., $\mu_{h}^{2} / \lambda_{h}>0$, can be easily satisfied since $\mu_{h}^{2}>0$ holds for most of the parameter space and $\lambda_{h}>0$ is guaranteed by the vacuum stability. However, a zero VEV for the singlet scalar will lead to a vanishing DM mass, which is of no interest to us in this work. To avoid such stationary points from being local minima, we can demand $\partial^{2} V / \partial s^{2}<0$ at these points, giving

$$
\mu_{s}^{2}+\frac{\lambda_{m} \mu_{h}^{2}}{2 \lambda_{h}}<0
$$

As we will see below, this condition also ensures a stationary point at $(h \neq 0, s \neq 0)$. 


\subsubsection{Stationary points along $s$-axis}

The stationary points along the $s$-axis is given by

$$
h=0, \quad s_{ \pm}=\frac{-\mu_{3} \pm \sqrt{\mu_{3}^{2}-4 \lambda_{s} \mu_{s}^{2}}}{2 \lambda_{s}} .
$$

The required physical condition is $\mu_{3}^{2}-4 \lambda_{s} \mu_{s}^{2}>0$. We find that for most of the parameter space having sufficiently strong first-order EWPT, the condition $\mu_{s}^{2}<0$ always holds. As we will see below, for the stationary points sitting on the $s$-axis, the potential located at $s_{+}$is always lower than the potential located at $s_{-}$if $\mu_{3}<0$ (i.e., $V\left(0, s_{+}\right)<V\left(0, s_{-}\right)$). On the other hand, $V\left(0, s_{+}\right)>V\left(0, s_{-}\right)$if $\mu_{3}>0$.

\subsubsection{Stationary points at $(h \neq 0, s \neq 0)$}

There are also solutions off the $h$-axis and $s$-axis, given by

$$
h=v, \quad s_{ \pm}=\frac{-\mu_{3} \pm \sqrt{\mu_{3}^{2}-4\left(\lambda_{s}-\frac{\lambda_{m}^{2}}{4 \lambda_{h}}\right)\left(\mu_{s}^{2}+\frac{\lambda_{m} \mu_{h}^{2}}{2 \lambda_{h}}\right)}}{2\left(\lambda_{s}-\frac{\lambda_{m}^{2}}{4 \lambda_{h}}\right)} .
$$

The condition for these solutions to be physical is

$$
\mu_{3}^{2}-4\left(\lambda_{s}-\frac{\lambda_{m}^{2}}{4 \lambda_{h}}\right)\left(\mu_{s}^{2}+\frac{\lambda_{m} \mu_{h}^{2}}{2 \lambda_{h}}\right)>0 .
$$

With vacuum stability condition (2.11), we have $\lambda_{s}-\frac{\lambda_{m}^{2}}{4 \lambda_{h}}>0$. As mentioned above, we can further impose the condition (2.13) on the potential if we demand that the stationary points along the $h$-axis be unstable. Hence, the condition (2.16) is satisfied for most of the parameter space of interest.

Using eq. (2.10), we obtain

$$
F \equiv V\left(h, s_{+}\right)-V\left(h, s_{-}\right)=-\frac{1}{6} \mu_{3}\left(s_{+}^{3}-s_{-}^{3}\right)-\frac{1}{4} \lambda_{s}\left(s_{+}^{4}-s_{-}^{4}\right) .
$$

One can show that $F<0$ is always true under the assumption of $\mu_{3}<0$. This is in fact equivalent to the condition

$$
\lambda_{s}>\frac{2 a\left(b^{2}-a c\right)}{3\left(b^{2}-2 a c\right)}, \quad \text { with } a=\lambda_{s}-\frac{\lambda_{m}^{2}}{4 \lambda_{h}}, b=\mu_{3}, \text { and } c=\mu_{s}^{2}+\frac{\lambda_{m} \mu_{h}^{2}}{2 \lambda_{h}} .
$$

Therefore, we finally reach the conclusion that with the assumption $\mu_{3}<0$, the tree-level potential located at $\left(h, s_{+}\right)$is always lower than the one located at $\left(h, s_{-}\right)$. Similarly, We can also prove that $F>0$ is always true provided $\mu_{3}>0$. We summarize our conclusion as follows:

$$
\left\{\begin{array}{l}
F<0 \text { and } w \equiv s_{+}>0 \text { if } \mu_{3}<0 \\
F>0 \text { and } w \equiv s_{-}<0 \text { if } \mu_{3}>0 .
\end{array}\right.
$$

One can easily verify that these conclusions are also established for the case of local minima along the $s$-axis. 
From eq. (2.8), we see that the pseudo-Goldstone DM mass is

$$
m_{\chi}^{2}=-3 \mu_{3} w
$$

To avoid a tachyonic mass for the DM candidate, the signs of $\mu_{3}$ and $w$ should be opposite. Without loss of generality, the singlet scalar's VEV of is assumed to be positive, and $\mu_{3}$ should thus have a negative value.

\subsection{Parameters}

Using eqs. (2.4), (2.9), and (2.10) we have

$$
\begin{aligned}
\mu_{h}^{2} & =\frac{1}{2} \mathcal{M}_{h h}^{2}(v, w)+\frac{w}{2 v} \mathcal{M}_{h s}^{2}(v, w) \\
\lambda_{h} & =\frac{\mathcal{M}_{h h}^{2}(v, w)}{2 v^{2}} \\
\lambda_{m} & =\frac{\mathcal{M}_{h s}^{2}(v, w)}{v w}, \\
\mu_{s}^{2} & =-\frac{1}{2} \mathcal{M}_{s s}^{2}(v, w)+\frac{1}{6} m_{\chi}^{2}-\frac{v}{2 w} \mathcal{M}_{h s}^{2}(v, w), \\
\mu_{3} & =-\frac{m_{\chi}^{2}}{3 w} \\
\lambda_{s} & =\frac{1}{2 w^{2}}\left(\mathcal{M}_{s s}^{2}(v, w)+\frac{1}{3} m_{\chi}^{2}\right) .
\end{aligned}
$$

The reason for employing eqs. (2.9) and (2.10) is to ensure the existence of a local minimum at $(v, w)$ for any choice of parameters. The above parameters can be related to three physical parameters, the masses of two Higgs bosons $m_{\mathcal{H}}$ and $m_{\mathcal{S}}$ and the mixing angle $\theta$, with the relations

$$
\begin{aligned}
& \mathcal{M}_{h h}^{2}(v, w)=\cos ^{2} \theta m_{\mathcal{H}}^{2}+\sin ^{2} \theta m_{\mathcal{S}}^{2}, \\
& \mathcal{M}_{s s}^{2}(v, w)=\sin ^{2} \theta m_{\mathcal{H}}^{2}+\cos ^{2} \theta m_{\mathcal{S}}^{2}, \\
& \mathcal{M}_{h s}^{2}(v, w)=\cos \theta \sin \theta\left(m_{\mathcal{S}}^{2}-m_{\mathcal{H}}^{2}\right),
\end{aligned}
$$

where $m_{\mathcal{H}}=125 \mathrm{GeV}$ and $v=246 \mathrm{GeV}$. Thus, we take $\left\{w, m_{\mathcal{S}}, m_{\chi}, \theta\right\}$ as the input parameters of the model.

\section{$3 \quad$ Effective potential}

At the one-loop level, the total effective potential is given by

$$
V_{\text {eff }}(h, s, T)=V(h, s)+V_{\mathrm{CW}}(h, s)+V_{T}(h, s, T)+V_{\mathrm{CT}}(h, s),
$$

where the tree-level potential $V(h, s)$ has been given above, and the other components are discussed below.

At zero temperature, the one-loop corrections to the potential is given using the $\overline{\mathrm{MS}}$ renormalization scheme [77] as

$$
V_{\mathrm{CW}}(h, s)=\frac{1}{64 \pi^{2}} \sum_{i} N_{i} M_{i}^{4}(h, s)\left[\log \frac{M_{i}^{2}(h, s)}{\mu^{2}}-C_{i}\right],
$$


where the subscript $i=\left\{\mathcal{H}, G, \mathcal{S}, \chi, Z_{\mathrm{T}}, Z_{\mathrm{L}}, W_{\mathrm{T}}, W_{\mathrm{L}}, t, b\right\}$ denote respectively the SM-like Higgs boson $\mathcal{H}$, SM Nambu-Goldstone bosons, heavy scalar $\mathcal{S}$, pseudo-Goldstone DM $\chi$, transverse and longitudinal components of SM gauge bosons, and top and bottom quarks, and $N_{i}=\{1,3,1,1,2,1,4,2,-12,-12\}$. The constant $C_{i}=1 / 2$ for gauge boson transverse modes and $3 / 2$ for all the other particles. The renormalization scale $\mu$ is set to be $v$ in this work. Appendix B gives the renormalization group equations (RGEs) for the parameters in the scalar potential, using which we calculate the renormalization group-improved (RGI) potential and show the dependence of the critical temperature and the corresponding VEV on the renormalization scale.

Due to plasma damping, the validity of the perturbative expansion of the effective potential breaks down at high temperatures. A remedy to this problem is to resum the daisy diagrams to all orders, which results in an additional contribution to the bosonic masses [78]. Thus, we replace the field-dependent bosonic masses at finite temperatures by

$$
M_{i}^{2}(h, s) \rightarrow M_{i}^{2}(h, s, T)=M_{i}^{2}(h, s)+\Pi_{i}(T),
$$

where the thermal corrections $\Pi_{i}(T)$ are given in appendix A. The one-loop potential becomes gauge-dependent when thermal corrections of bosons' masses are introduced [79, 80], leading to gauge-dependent critical temperature and GW spectrum produced from phase transition [34, 81]. To focus on our topic, in this work we take the Landau gauge with a vanishing gauge-fixing parameter $(\xi=0)$ for the effective potential. In section 9 we will scrutinize the gauge dependence issue. There we will show that our main conclusions made in the following sections with the $\xi=0$ effective potential are not changed when compared with the analyses made by using a gauge-independent effective potential proposed in ref. [82].

At the one-loop level, the finite-temperature contributions to the effective potential are given by $[76,83]$

$$
\begin{aligned}
V_{\mathrm{T}}(h, s, T) & =\frac{T^{4}}{2 \pi^{2}} \sum_{i} N_{i} J_{B, F}\left(M_{i}^{2}(h, s, T) / T^{2}\right), \\
\text { where } \quad J_{B, F}\left(z^{2}\right) & =\int_{0}^{\infty} d x x^{2} \ln \left(1 \mp e^{-\sqrt{x^{2}+z^{2}}}\right),
\end{aligned}
$$

with the - sign for bosons and + for fermions.

To maintain the main properties of the tree-level potential derived above, we add the following counter-terms to the potential at zero temperature

$$
V_{\mathrm{CT}}(h, s)=-\frac{1}{2} \delta \mu_{h}^{2} h^{2}+\frac{1}{4} \delta \lambda_{h} h^{4}+\frac{1}{4} \delta \lambda_{m} h^{2} s^{2}+\frac{1}{2} \delta \mu_{s}^{2} s^{2}+\frac{1}{3} \delta u_{3} s^{3}+\frac{1}{4} \delta \lambda_{s} s^{4} .
$$

The coefficients of the counter-term potential are given in appendix C.

\section{Parameter space for electroweak phase transition}

In this section, we scan the parameter space for viable sample points for a sufficiently strong first-order phase transition. We also show the distributions of the model parameters and physical parameters based upon our scan results. 

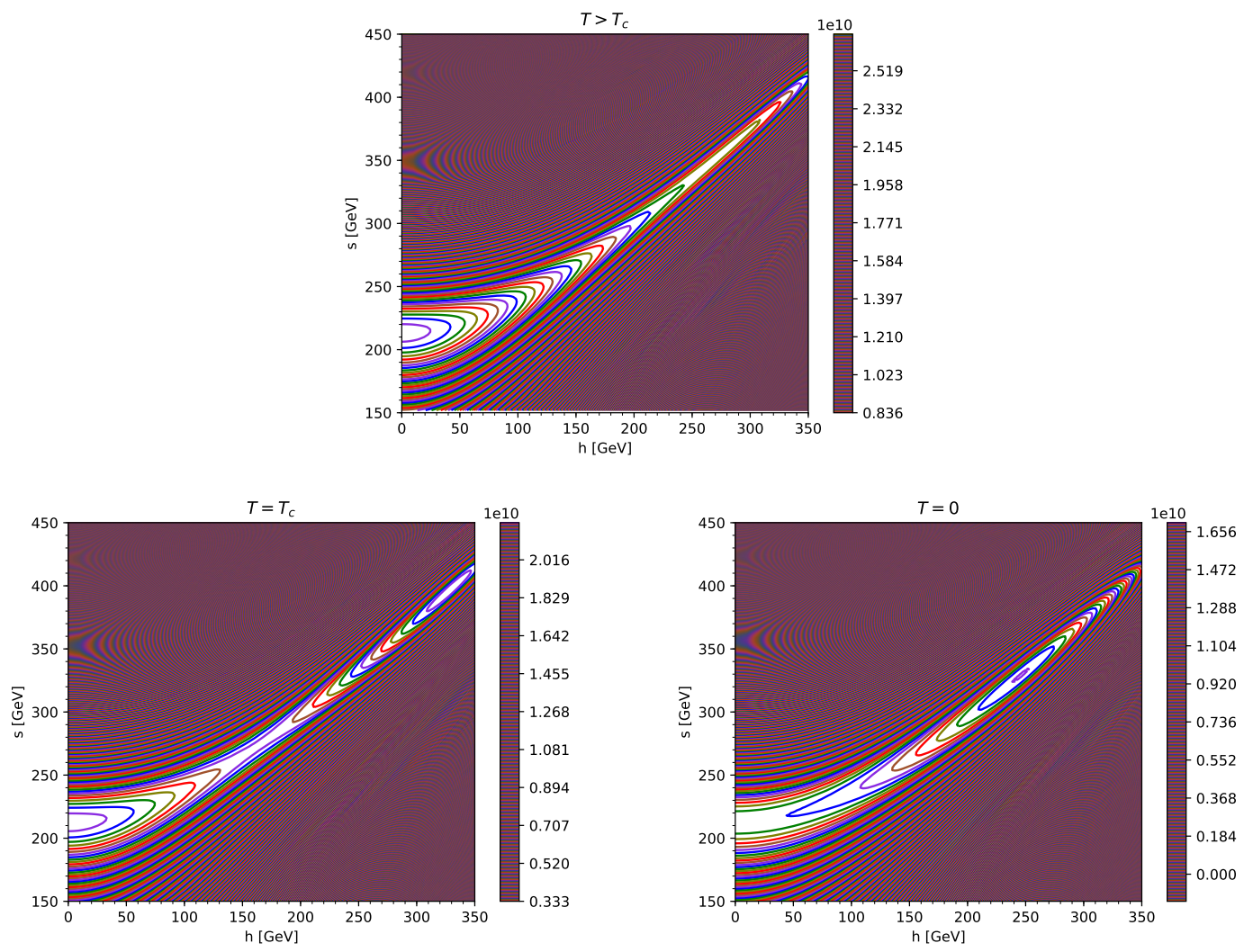

Figure 1. Contours of total effective potential in the $h-s$ plane, with parameters $w=329.2 \mathrm{GeV}$, $m_{\mathcal{S}}=702.0 \mathrm{GeV}, m_{\chi}=137.1 \mathrm{GeV}$, and $\theta=-0.64$. From upper plot to lower right plot, the potential is evaluated at temperature $T>T_{c}, T=T_{c}$, and $T=0$.

\subsection{Two-step phase transition}

A strong first-order EWPT could occur if there is a sufficiently high and wide potential barrier separating the two degenerate vacua of the thermal effective potential at critical temperature. Introducing an extra bosonic degree of freedom could enhance the barrier and thus make the EWPT stronger. In our model, there are two contributions to the barrier in the effective potential [30]: one is the tree-level barrier coming from the cubic term of the tree-level potential; the other one arises from the bosonic thermal corrections to the potential at the one-loop level. To see the latter, one can expand the integrations (3.4) in the high temperature limit, i.e., $z \equiv M_{i}^{2}(h, s) / T^{2} \ll 1$, and find that there is a term proportional to $z^{3 / 2}$ that leads to terms cubic in both $h$ and $s$. Most importantly, the barrier term cubic in $h$ is proportional to the Higgs portal coupling $\lambda_{m}$, and thus to the mixing angle $\theta$ (see eq. (2.23)) [48, 84-86]. Therefore a large value of mixing angle could strengthen the EWPT by boosting the potential barrier. The potential barrier is shallow in the SM and its EWPT is confirmed to be a crossover [9].

In this work, we focus on the so-called two-step phase transition as shown in figure 1. At very high temperatures, both scalar fields have no VEV. When the temperature drops 
to a certain value $T_{s}$, (above the critical temperature $T_{c}$, at which two degenerate vacua exist concurrently) the scalar potential along the $s$ direction develops a global minimum at $\left(0, w_{0}\left(T_{s}\right)\right)$. We call it the symmetric phase, as shown in the upper plot of figure 1 . As the temperature further lowers to the critical temperature, another local minima located at $\left(v\left(T_{c}\right), w\left(T_{c}\right)\right)$, designating the symmetry-broken phase, appears and becomes degenerate with the symmetric phase $\left(0, w_{0}\left(T_{c}\right)\right)$, as shown in the lower left plot of figure 1 . Meanwhile, a tunneling path, along which is a lowest barrier between the two degenerate minima, opens up. The decrease of potential at the broken phase is much faster than that at the symmetric phase as the temperature approaches zero. As a result, the broken phase moves to $(v, w)$ and becomes a global minimum, as shown by the lower right plot of figure 1 .

\subsection{Searching scheme}

The strength of the EWPT is measured according to the order parameter $v_{c} / T_{c}$, where $v_{c} \equiv v\left(T_{c}\right)$ is the VEV of SM-like Higgs boson at the critical temperature. For a successful baryogenesis, the first-order EWPT should be strong enough so that the sphaleron process in the broken phase is sufficiently suppressed to avoid baryon asymmetry washout [8]. This gives the conventional criterion for a sufficiently strong EWPT:

$$
\frac{v_{c}}{T_{c}} \gtrsim \zeta
$$

where $\zeta$ is a criterion value usually around unity. We note that a theoretical ambiguity of this criterion may arise due to the gauge dependence and uncertainty from higherorder calculations, as studied in ref. [80]. Nevertheless, we will still use it as a useful guidance to the relevant regions in the parameter space. For the left-hand side (1.h.s.) of eq. (4.1), the ratio $v_{c} / T_{c}$ obtained by using our procedure provided below is gaugedependent. As mentioned above, the full one-loop effective potential is gauge-dependent due to the thermal corrections. With the leading-order high-temperature expansion of the effective potential and the implementation of Nielsen's identity, ref. [80] provided a gaugeindependent determination of $v_{c}, T_{c}$ and thus $v_{c} / T_{c}$. To estimate the impacts of gauge dependence in criterion (4.1), we will adopt the gauge-independent effective potential, which is obtained by truncating the one-loop effective potential at second order in the EW gauge couplings, to calculate $v_{c} / T_{c}$ in section 9 . We will show that in comparison with the full one-loop potential, using the gauge-independent potential generally reduces the critical temperature while increasing $v_{c} / T_{c}$. Thus, the samples satisfying criterion (4.1) in the Landau gauge can also satisfy the gauge-independent version of criterion (4.1). For the r.h.s. of criterion (4.1), there exist several sources of theoretical uncertainties in obtaining this quantity (a summary of these uncertainties can be found in ref. [80] and references therein). One of the uncertainties is the lower bound on the "washout factor," $S>e^{-X}$, where $S$ is the ratio of the baryon densities after and before the phase transition. Taking $X \simeq 10$ could lead to the conventional criterion value, $\zeta \simeq 1.0$. However, for a certain scenario, uncertainties in the value of $X$ could arise from the efficiency of the CP violation mechanism and the duration of phase transition. A more realistic treatment of the criterion is to replace unity by a range determined by an appropriate choice of $X$ and 
the other theoretical inputs [80]. In this work, we will simply take the conventionally used criterion value $\zeta \simeq 1.0$ for the determination of a sufficiently strong phase transition.

Aiming at the search of parameter space giving a sufficiently strong EWPT, we make a random scan of the parameters in the following ranges:

$$
\begin{aligned}
100 \mathrm{GeV} & \leq w \leq 2000 \mathrm{GeV}, & 150 \mathrm{GeV} & \leq m_{\mathcal{S}} \leq 2000 \mathrm{GeV}, \\
10 \mathrm{GeV} & \leq m_{\chi} \leq 1500 \mathrm{GeV}, & -\frac{\pi}{4} & \leq \theta \leq \frac{\pi}{4} .
\end{aligned}
$$

In addition to the above enforced restrictions, the parameters are also subject to the constraints discussed in section 2.2, including vacuum stability (2.11) and the conditions to ensure the existence of stationary points at the symmetric phase $\left(0, w_{0}\right)$ and the broken phase $(v, w)$. Further constraints come from the requirement of perturbative unitarity $[76,87]$

$$
\lambda_{h}<4 \pi, \lambda_{s}<4 \pi,\left|\lambda_{m}\right|<8 \pi, 3 \lambda_{h}+2 \lambda_{s}+\sqrt{\left(3 \lambda_{h}-2 \lambda_{s}\right)^{2}+2 \lambda_{m}^{2}}<8 \pi .
$$

We also require that $V(v, w)<V\left(0, w_{0}\right)$ and $V(v, w)<V(0,0)$ at zero temperature, so that the broken phase is a global minimum.

To search for the critical temperature where two degenerate vacua coexist, we start from an initial temperature $T$ between a minimum value of temperature $T_{\min }$ and a maximum value of temperature $T_{\max }$, we then search between these two temperatures for the local minima of the potential around the positions $\left(0, w_{0}\right)$ and $(v, w)$, which can be determined using the analytical formulas (2.14) and (2.15) for given parameters. If the local minimum at the symmetric phase $\left(0, w_{0}(T)\right)$ is found to be larger (smaller) than the one at the broken phase $(v(T), w(T))$, the temperature is increased (decreased) in the next trial. We conclude that no electroweak phase transition for given parameters occurs if the following two cases are met:

- Case 1 . The local minimum at the symmetric phase $\left(0, w_{0}\left(T_{\max }\right)\right)$ is larger than the one at the broken phase $\left(v\left(T_{\max }\right), w\left(T_{\max }\right)\right)$.

- Case 2. The local minimum at the symmetric phase $\left(0, w_{0}\left(T_{\min }\right)\right)$ is less than the one at the broken phase $\left(v\left(T_{\min }\right), w\left(T_{\min }\right)\right)$.

As obvious, the lower and upper temperature limits are critical for our parameters searches. In our preliminary scan of the parameter space, we find that no points with $v_{c} / T_{c} \gtrsim 1$ can be found at a temperature higher than about $350 \mathrm{GeV}$. We thus restrict the scan range of temperature to $(10-350) \mathrm{GeV}$.

\subsection{Parameter distributions}

We generate one million random floats uniformly for each of the input parameters, among which about $1.8 \%$ are found to be able to trigger a sufficiently strong phase transition while fulfilling the other basic criteria mentioned above. We show the distributions of various physical parameters in figure 2, and summarize our observations from the figures as follows: 

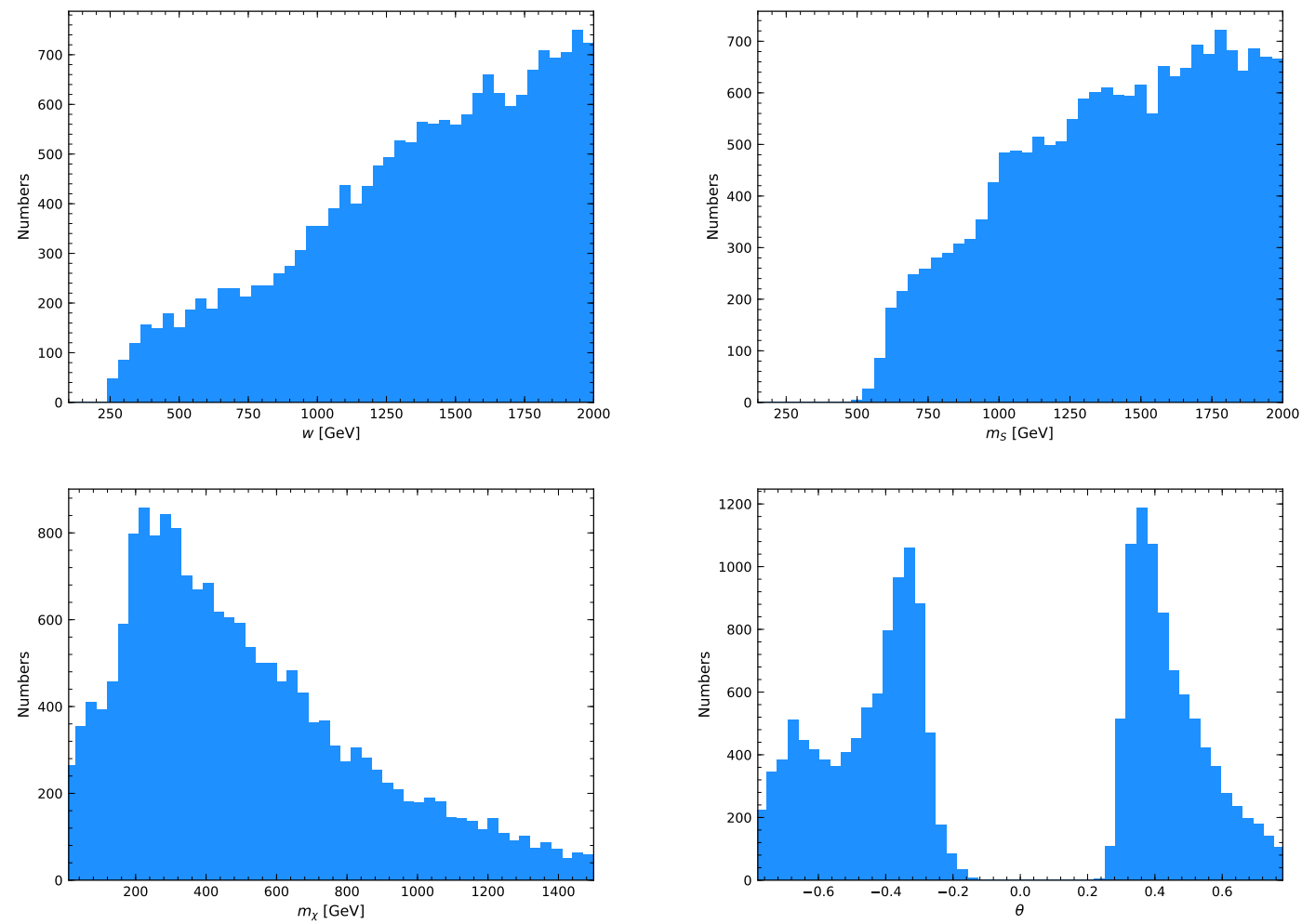

Figure 2. Distributions of parameters that can generate a sufficiently strong first-order EWPT. For each fixed value of the parameter on the horizontal axis, all the other parameters are scanned in their respective full ranges.

1. There is a lower bound in the distribution of $m_{\mathcal{S}}$, i.e., $m_{\mathcal{S}} \gtrsim 500 \mathrm{GeV}$. This is because in order to trigger a phase transition, the local minimum at the broken phase $(v(T), w(T))$ should be larger than the local minimum at the symmetric phase $\left(0, w_{0}(T)\right)$ when the temperature is higher than critical temperature. To see this, we show in the upper left plot of figure 3 the potential difference $\Delta V_{b s}(T) \equiv$ $V_{\text {eff }}(v(T), w(T), T)-V_{\text {eff }}\left(0, w_{0}(T), T\right)$ at temperature $T=300 \mathrm{GeV}$ (the distribution of critical temperature can be found in figure 4) as a function of $m_{\mathcal{S}}$, with $\theta=0.4$ and $m_{\chi}=300 \mathrm{GeV}$. The blue, green, and red points in figure 3 represent the results with $w=300 \mathrm{GeV}, 400 \mathrm{GeV}$, and $500 \mathrm{GeV}$, respectively. As shown in the drawing, the potential difference slowly increases with $m_{\mathcal{S}}$ and remains negative for $m_{\mathcal{S}} \lesssim 500 \mathrm{GeV}$. At larger values of $m_{\mathcal{S}}$, the potential difference sharply increases to a positive value. Therefore, the lower bound $m_{\mathcal{S}} \gtrsim 500 \mathrm{GeV}$ is to guarantee a successful phase transition.

2. There is a lower bound in the distribution of $|\theta|$, i.e., $|\theta| \gtrsim 0.2$. Such a relatively large lower bound on the absolute value of the mixing angle is straightforwardly derived from the requirement of a sufficiently strong first-order EWPT. As shown in eq. (2.23), the mixing angle $|\theta|$ directly controls the Higgs-portal interacting strength 

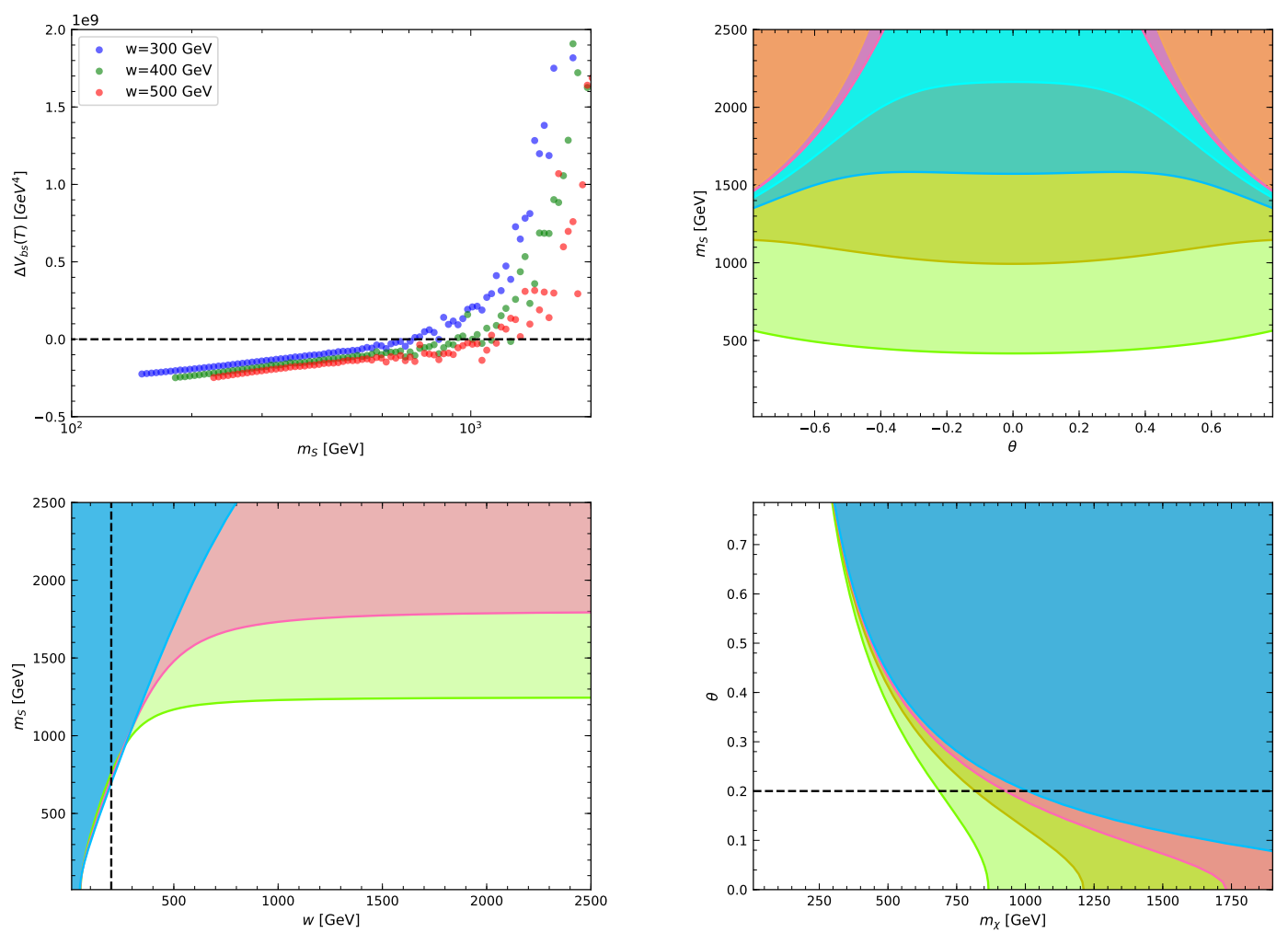

Figure 3. Upper left plot: $\Delta V_{b s}(T)$ as a function of $m_{\mathcal{S}}$, with $\theta=0.4, m_{\chi}=300 \mathrm{GeV}$, and $T=300 \mathrm{GeV}$. The blue, green, and red points represent respectively the results with $w=300 \mathrm{GeV}$, $400 \mathrm{GeV}$, and $500 \mathrm{GeV}$. Upper right plot: unitarity bounds in the $\theta-m_{\mathcal{S}}$ plane, with $m_{\chi}=300 \mathrm{GeV}$. Colored regions in this figure are excluded by various constraints. The values of $w$ in this plot are $100 \mathrm{GeV}$ (green), $200 \mathrm{GeV}$ (yellow), $300 \mathrm{GeV}$ (deep blue), $400 \mathrm{GeV}$ (light blue), $600 \mathrm{GeV}$ (pink), and $1000 \mathrm{GeV}$ (sandy-brown) respectively. Lower left plot: unitarity bounds in the $w$ - $m_{\mathcal{S}}$ plane, with $\theta=0.2$ (blue), 0.4 (pink), and 0.6 (green), respectively. Lower right plot: condition (2.13) in the $m_{\chi^{-}} \theta$ plane, with $w=1000 \mathrm{GeV}$. The green, yellow, pink, and blue regions represent the bounds with $m_{\mathcal{S}}=500 \mathrm{GeV}, 700 \mathrm{GeV}, 1000 \mathrm{GeV}$, and $1500 \mathrm{GeV}$, respectively.

$\left|\lambda_{m}\right|$ (whose distribution can be found in the left plot of the second row of figure 4). For $|\theta| \lesssim 0.2$ (or, equally, $\left|\lambda_{m}\right| \lesssim 1$ ), the Higgs boson $h$ and singlet scalar $s$ have nearly no mixing and the phase transition takes place mostly along the $h$ direction. A larger $|\theta|$ would induce a significant mixing between the $h$ and $s$ fields and curve the tunneling path to be along a linear combination of the two fields (see figure 1) and finally lead to a strong EWPT (see also ref. [88] for similar conclusions).

3. The lower bound on $w$ and the decrease in the distribution of $|\theta|$ at larger values are from the bound of unitarity (last condition of eq. (4.3)). We plot this bound condition in various planes in figure 3 , the colored regions are excluded by the constraints. In the upper right plot of figure 3 , we show the unitarity bounds in the $\theta-m_{\mathcal{S}}$ plane, fixing $m_{\chi}=300 \mathrm{GeV}$. The values of $w$ in this plot are $100 \mathrm{GeV}$ (green), $200 \mathrm{GeV}$ (yellow), $300 \mathrm{GeV}$ (blue), $400 \mathrm{GeV}$ (light blue), $600 \mathrm{GeV}$ (pink), and $1000 \mathrm{GeV}$ (sandy-brown), 
respectively. As shown in this plot, when $w=100 \mathrm{GeV}$, the heavy scalar mass $m_{\mathcal{S}}$ is restricted to lie below about $500 \mathrm{GeV}$. As one increases $w$, more parameter space opens up. Sufficient sample points for strong EWPT are available for $w \gtrsim 200 \mathrm{GeV}$. These indicate that the unitarity bound on large values of $m_{\mathcal{S}}$ can be avoided by increasing $w$. In the lower left plot of figure 3, we plot the unitarity bounds in the $w$ - $m_{\mathcal{S}}$ plane, with $\theta=0.2$ (blue), 0.4 (pink), and 0.6 (green), respectively. As shown in this plot, the available parameter space is largely reduced with the increase mixing angle, which explains the decreasing behavior in the $|\theta|$ distribution at large values. When $m_{\mathcal{S}} \gtrsim 500 \mathrm{GeV}$, nearly all of the parameter space is excluded by the unitarity bound for $w \lesssim 200 \mathrm{GeV}$. The constraints become independent of $m_{\mathcal{S}}$ for $w \gtrsim 500 \mathrm{GeV}$ and $\theta \gtrsim 0.4$.

4. The distribution of pseudo-Goldstone DM mass $m_{\chi}$ has a peak around (200-400) GeV and then decreases at larger values. The decreasing behavior of $m_{\chi}$ distribution involves a few contributions. One is the unitarity bound; it is easy to check that the unitarity bound becomes tighter for a larger DM mass $m_{\chi}$. Another reason is the condition (2.13), which is to ensure that the stationary point located along the $h$ axis cannot be a local minimum. We show this constraints in the lower right plot of figure 3 , the colored regions are excluded by the constraints. In this plot, the value of $w$ is fixed at $1000 \mathrm{GeV}$, the green, yellow, pink, and blue regions represent the bounds with $m_{\mathcal{S}}=500 \mathrm{GeV}, 700 \mathrm{GeV}, 1000 \mathrm{GeV}$, and $1500 \mathrm{GeV}$, respectively. We see that for $\theta \gtrsim 0.2$, the parameter space with $m_{\chi} \gtrsim 1000 \mathrm{GeV}$ is excluded. However, we should note that this bound is imposed only when there is a stationary point along $h$ axis, which requires $\mu_{h}^{2}>0$. From the distribution of $\mu_{h}^{2}$ shown in the upper left plot of figure 4 , we find that only about half of the total sample points have a stationary point along $h$ axis. We also note that such a requirement is somehow too stringent because of the loop corrections on the potential. Further constraint may arise from the requirement of $d \Delta V_{b s}(T) / d T^{2}>0$ near the critical temperature [75], we find this bound is relatively weak and do not give a detailed account here.

5. We see that within the parameter ranges considered here, the distributions of $w$ and $m_{\mathcal{S}}$ do not decrease with increasing values. Such distributions are consistent with a recent global fit performed in ref. [32].

In figure 4 we show the distributions of the various parameters in the scalar potential, which are determined from the four physical parameters above using eqs. (2.21)-(2.26). The Higgs self coupling $\lambda_{h}$ takes a value between 1 and 4 most of the time. The mixing coupling $\lambda_{m}$ can take either sign, but cannot be too small, as demanded by $|\theta| \gtrsim 0.2$. Due to our choice of a positive $w, \mu_{3}$ takes only negative values in order to have a positive DM mass, given in eq. (2.20). The self coupling parameter of the $S$ field is usually less than 1 , though larger values are sometimes allowed as well. The distributions of the critical temperature $T_{c}$ and the ratio $v_{c} / T_{c}$ are also depicted in the figure, with $T_{c}$ falling mostly between 150 and $300 \mathrm{GeV}$. 

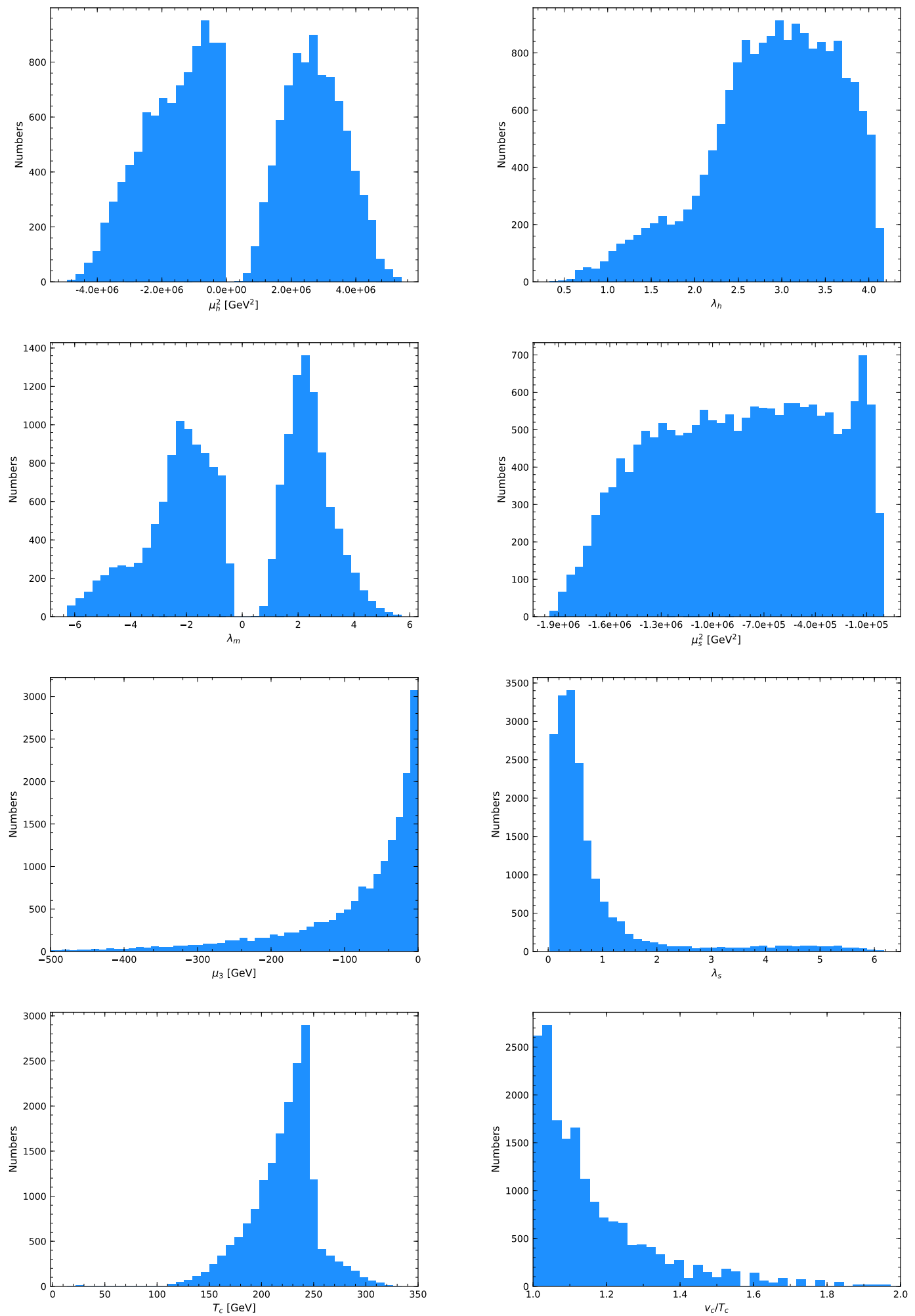

Figure 4. Distributions of parameters in the effective potential that can generate a sufficiently strong first-order EWPT. The lowest two plots give the distributions of $v_{c} / T_{c}$ and $T_{c}$. 


\section{Vacuum stability and perturbativity}

In this work, we restrict ourselves in the case of absolute vacuum stability, which requires that the EW vacuum of the scalar effective potential at zero temperature be a global minimum below the energy scale for which the model is valid.

For our model, the tree-level vacuum stability is implemented in eq. (2.11). For the vacuum stability at one-loop level, we follow the gauge-invariant treatment used in ref. [17] by requiring

$$
\lambda_{h}(\mu)>0, \quad \lambda_{s}(\mu)>0, \quad \lambda_{h}(\mu) \lambda_{s}(\mu)>\frac{1}{4} \lambda_{m}(\mu)^{2}, \text { for } \mu<\Lambda,
$$

where $\Lambda$ is a cutoff energy scale where new physics comes in or perturbation breaks down. These extend the requirements of tree-level vacuum stability on the couplings to any energy scale below the cutoff scale. These conditions ensure a positive potential for large field values. The large-field behavior of the potential is dominated by the quartic terms. With the relations given in eq. (2.21), the quartic part of the potential is given by [75]

$$
V_{4}=\frac{1}{8 \mathcal{M}_{h}^{2} v^{2}}\left[\left(\mathcal{M}_{h}^{2} h^{2}+\lambda_{m} v^{2} s^{2}\right)^{2}+4 \lambda^{2} v^{4} s^{4}\right]
$$

where $\lambda^{2} \equiv \lambda_{h} \lambda_{s}-\lambda_{m}^{2} / 4$. We see that if $\lambda_{m}<0$, the first term in the square brackets vanishes along a particular direction. In this case, the last condition of eq. (5.1) ensures the stability of the EW vacuum. However, in the case of $\lambda_{m}>0$, the condition $\lambda^{2}>0$ could be over-restrictive since the potential is already bounded from below for large values of the fields along all directions with the first two conditions of eq. (5.1).

We now turn to the perturbativity of the scalar potential. At the one-loop level, a large Higgs quartic coupling at EW scale may give rise to a positive $\beta_{\lambda_{h}}$ function, which results in a monotonic increase of $\lambda_{h}(\mu)$ with $\mu$ and eventually develops a Landau pole at some scale $\Lambda_{\mathrm{L}}$. However, the perturbativity of theory has already been invalid long before $\mu$ reaches $\Lambda_{\mathrm{L}}$. At the two-loop level, $\lambda_{h}(\mu)$ may approach an ultraviolet fixed point where $\beta_{\lambda_{h}} \rightarrow 0$ and $\lambda_{h}(\mu) \rightarrow \lambda_{\mathrm{FP}}$ [89]. For a given cutoff scale $\Lambda$, perturbation theory is expected to be reliable for the value of $\lambda_{\Lambda} \equiv \lambda_{h}(\Lambda)$ in the range of $\lambda_{\mathrm{FP}} / 4-\lambda_{\mathrm{FP}} / 2$ [90]. Following ref. [17], the approximate perturbativity bounds on the couplings are given by

$$
\lambda_{h}(\mu)<\lambda_{\Lambda}, \quad \lambda_{s}(\mu)<\lambda_{\Lambda}, \quad \lambda_{m}(\mu)<\lambda_{\Lambda}, \quad \text { for } \mu<\Lambda .
$$

Given the cutoff scale $\Lambda$, we evolve the running couplings from the EW scale up to $\Lambda$ with the RG equations. We pick out those samples simultaneously satisfying the constraints from both vacuum stability and perturbativity, and present the results in figure 5 . In the figure, we show the distributions of $\theta, \lambda_{h}$, and $\lambda_{s}$ with various choices of $\Lambda$ and $\lambda_{\Lambda}$. The constraints, of course, become stronger with a larger $\Lambda$ and a lower $\lambda_{\Lambda}$. We find that most of the samples satisfy the vacuum stability up to a very large scale, while perturbativity can impose a stricter bound on the parameters. For the case of $\lambda_{\Lambda}=\lambda_{\mathrm{FP}} / 2$, the constraints with $\Lambda=10^{3} \mathrm{GeV}$ (light green histogram) are nearly negligible so that its distribution overlaps with the initial distribution (blue histogram). We see that in this case, most of 

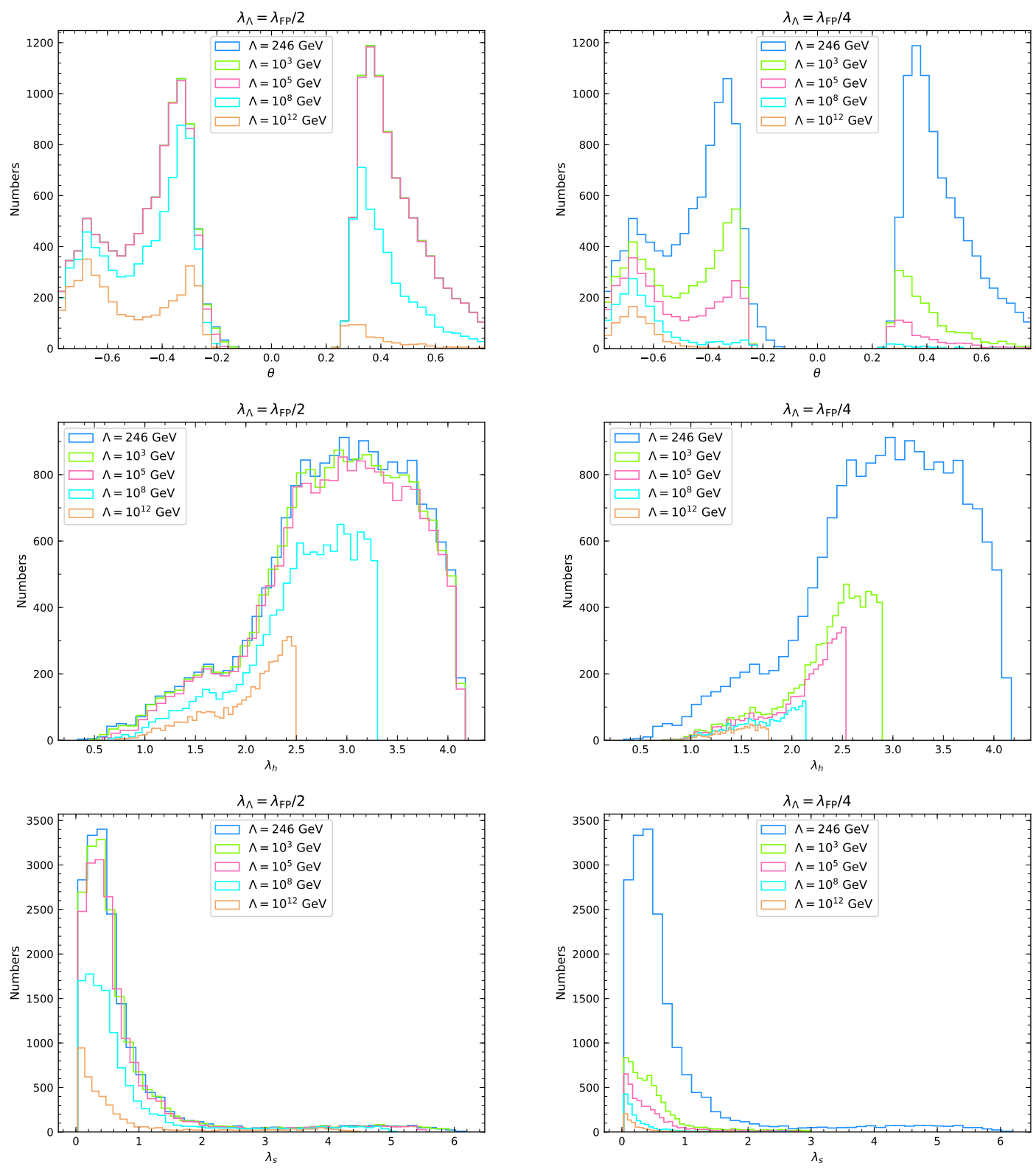

Figure 5. Distributions of parameters after taking into account the constraints from vacuum stability and perturbativity at the one-loop level. The blue, green, pink, light blue, and yellow histograms represent the bounds with $\Lambda=246 \mathrm{GeV}, 10^{3} \mathrm{GeV}, 10^{5} \mathrm{GeV}, 10^{8} \mathrm{GeV}$, and $10^{12} \mathrm{GeV}$, respectively. $\lambda_{\Lambda}=\lambda_{\mathrm{FP}} / 2$ for the left plots and $\lambda_{\mathrm{FP}} / 4$ for the right plots.

the parameter space of the theory remains valid for the energy scale below $\sim 10^{8} \mathrm{GeV}$. However, for the case of $\lambda_{\Lambda}=\lambda_{\mathrm{FP}} / 4$, most of the samples are excluded for the scale above $\sim 10^{5} \mathrm{GeV}$. We note that the constraints from vacuum stability and perturbativity are subjective due to the somewhat arbitrary choice of the cutoff scale and the uncertainties in the value of $\lambda_{\Lambda}[17]$. 


\section{Experimental constraints}

In this section, we consider various constraints of collider experiments on our model, including the electroweak precision observable measurements and Higgs signal strength measurements.

\subsection{Electroweak precision observables}

We first consider the constraints arising from electroweak precision observables (EWPOs) [91, 92]. We introduced two scalars to the SM, one of them is a real singlet scalar which can mix with the SM Higgs boson. Both the SM Higgs boson and the real singlet scalar will contribute to the SM gauge boson self-energies at loop-level and finally induce corrections to the oblique parameters $S, T$, and $U$. The other one is a pseudo-Goldstone boson $\chi$ which becomes a DM candidate due to an accidental $\mathbb{Z}_{2}$ symmetry hidden in our $\mathbb{Z}_{3}$ symmetry model. The pseudo-Goldstone boson do not mix with SM particles and thus do not affect the oblique parameters. Constraints from the EWPO's can alter the distribution of $m_{\chi}$ via the parameters $m_{\mathcal{S}}$ and $\theta$.

The contributions of the heavy scalar to the oblique parameters $\Delta \mathcal{O}_{i} \equiv \mathcal{O}_{i}-\mathcal{O}_{i}^{\mathrm{SM}}$ (here $i$ denotes $T, S$, or $U$ ) can be quantified as follows [28, 48]:

$$
\begin{aligned}
\Delta T= & \frac{3}{16 \pi s_{W}^{2}}\left[\cos ^{2} \theta\left\{f_{T}\left(\frac{m_{\mathcal{H}}^{2}}{m_{W}^{2}}\right)-\frac{1}{c_{W}^{2}} f_{T}\left(\frac{m_{\mathcal{H}}^{2}}{m_{Z}^{2}}\right)\right\}+\sin ^{2} \theta\left\{f_{T}\left(\frac{m_{\mathcal{S}}^{2}}{m_{W}^{2}}\right)\right.\right. \\
& \left.\left.-\frac{1}{c_{W}^{2}} f_{T}\left(\frac{m_{\mathcal{S}}^{2}}{m_{Z}^{2}}\right)\right\}-\left\{f_{T}\left(\frac{m_{\mathcal{H}}^{2}}{m_{W}^{2}}\right)-\frac{1}{c_{W}^{2}} f_{T}\left(\frac{m_{\mathcal{H}}^{2}}{m_{Z}^{2}}\right)\right\}\right], \\
\Delta S= & \frac{1}{2 \pi}\left[\cos ^{2} \theta f_{S}\left(\frac{m_{\mathcal{H}}^{2}}{m_{Z}^{2}}\right)+\sin ^{2} \theta f_{S}\left(\frac{m_{\mathcal{S}}^{2}}{m_{Z}^{2}}\right)-f_{S}\left(\frac{m_{\mathcal{H}}^{2}}{m_{Z}^{2}}\right)\right], \\
\Delta U= & \frac{1}{2 \pi}\left[\cos ^{2} \theta f_{S}\left(\frac{m_{\mathcal{H}}^{2}}{m_{W}^{2}}\right)+\sin ^{2} \theta f_{S}\left(\frac{m_{\mathcal{S}}^{2}}{m_{W}^{2}}\right)-f_{S}\left(\frac{m_{\mathcal{H}}^{2}}{m_{W}^{2}}\right)\right]-\Delta S,
\end{aligned}
$$

where $m_{Z}$ and $m_{W}$ are the SM weak gauge boson masses, the cosine of the weak mixing angle $c_{W}^{2}=m_{W}^{2} / m_{Z}^{2}$ and $s_{W}^{2}=1-c_{W}^{2}$. The loop functions $f_{T}(x)$ and $f_{S}(x)$ are given by $[32,93]$

$$
\begin{aligned}
& f_{T}(x)=\frac{x \log x}{x-1}, \\
& f_{S}(x)=\left\{\begin{aligned}
\frac{1}{12}[ & -2 x^{2}+9 x+\left((x-3)\left(x^{2}-4 x+12\right)+\frac{1-x}{x}\right) f_{T}(x) \\
& \left.+2 \sqrt{(4-x) x}\left(x^{2}-4 x+12\right) \tan ^{-1} \sqrt{\frac{4-x}{x}}\right], \text { for } 0<x<4, \\
\frac{1}{12}[ & -2 x^{2}+9 x+\left((x-3)\left(x^{2}-4 x+12\right)+\frac{1-x}{x}\right) f_{T}(x) \\
& \left.+\sqrt{(x-4) x}\left(x^{2}-4 x+12\right) \log \left(\frac{x-\sqrt{(x-4) x}}{x+\sqrt{(x-4) x}}\right)\right], \text { for } x \geq 4 .
\end{aligned}\right.
\end{aligned}
$$




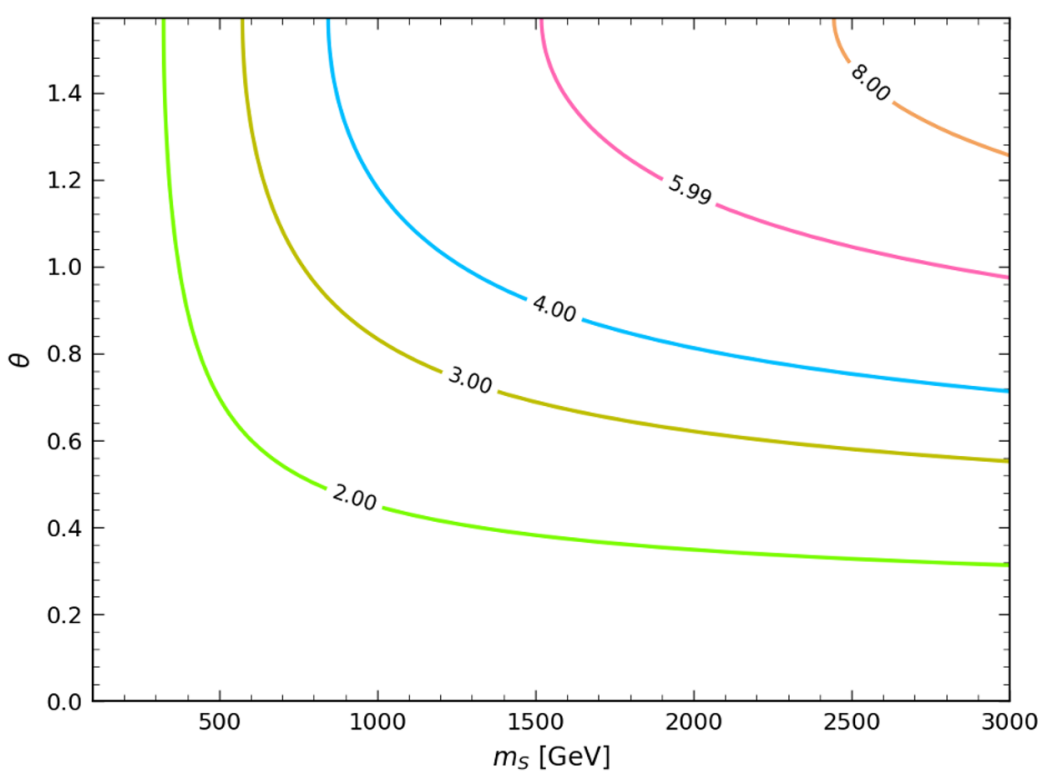

Figure 6. Contours of $\Delta \chi_{\text {ewpo }}^{2}$ in the $m_{\mathcal{S}}-\theta$ plane.

In order to analyze the impacts of the EWPO's on the strong EWPT parameter distributions, we follow the procedure given in refs. [48, 55, 88, 94] by defining a $\Delta \chi^{2}$ as

$$
\Delta \chi_{\text {ewpo }}^{2}=\sum_{i, j}\left(\Delta \mathcal{O}_{i}-\Delta \mathcal{O}_{i}^{\exp }\right)\left(\Sigma^{2}\right)_{i j}^{-1}\left(\Delta \mathcal{O}_{j}-\Delta \mathcal{O}_{j}^{\exp }\right),
$$

where $\Delta \mathcal{O}_{i}^{\exp }$ denotes the experimental measurements of the deviations of oblique parameters from its SM reference values. We take the most recent analysis from the Gfitter Group [95]

$$
\Delta S=0.04 \pm 0.11, \quad \Delta T=0.09 \pm 0.14, \quad \Delta U=-0.02 \pm 0.11
$$

The covariance matrix $\Sigma_{i j}^{2} \equiv \sigma_{i} \rho_{i j} \sigma_{j}$ involves $\sigma_{i}$ as the various errors given in eqs. (6.4) and

$$
\rho_{i j}=\left(\begin{array}{ccc}
1 & 0.92 & -0.68 \\
0.92 & 1 & -0.87 \\
-0.68 & -0.87 & 1
\end{array}\right)
$$

is the correlation matrix of the experiment. The electroweak observables are governed by only two parameters: the heavy scalar mass $m_{\mathcal{S}}$ and the mixing angle $\theta$. We consider the singlet scalar extended models to be consistent with the EWPO's if the oblique parameters lie within the $95 \%$ confidence level (CL) ellipsoid, which corresponds to taking $\Delta \chi_{\text {ewpo }}^{2} \leq$ 5.99 for the models with given $m_{\mathcal{S}}$ and $\theta$.

We plot the contours of $\Delta \chi_{\text {ewpo }}^{2}$ in figure 6 and find that in the $m_{\mathcal{S}}-\theta$ space, the region with $m_{\mathcal{S}} \gtrsim 1500 \mathrm{GeV}$ and $\theta \gtrsim 1.0$ (to the right of the pink curve) is excluded at the $95 \%$ CL. The constraint is seen to be not very strong. 


\subsection{Higgs signal strengths}

In our extension of the SM with a complex singlet scalar, the SM-like Higgs boson coupling strengths to other SM particles are modified by a common factor of $\cos \theta$. This leads to the productions of $X \bar{X}$ (where $X$ can be a SM gauge boson or fermion) via the SM-like Higgs boson being suppressed by a factor $\cos ^{2} \theta$, provided that no additional decay channel is permitted. Note that the same factor of $\cos ^{2} \theta$ is involved in all major production channels (gluon fusion, vector boson fusion and $t \bar{t} h$ ). To take into account the published exclusion bounds from Higgs searches at the LEP, Tevatron and LHC experiments, we make use of the HiggsBounds_v4.3.1 [96] package, which calculates the predicted signal rates for the search channels considered in the experimental data. More information on search channels and experimental data used in HiggsBounds can be found in ref. [96]. The HiggsBounds package determines whether a point in the model parameter space is excluded at 95\% CL by comparing the predicted signal rates against the expected and observed cross section limits from the direct Higgs searches [96].

Distinct signal strengths, defined as the production rate times the decay branching fraction relative to the $\mathrm{SM}$ expectation, i.e., $\mu_{i} \equiv(\sigma \times \mathrm{BR})_{i} /(\sigma \times \mathrm{BR})_{i}^{\mathrm{SM}}$, in various decay channels including $\gamma \gamma, W W^{*}, Z Z^{*}, b \bar{b}$, and $\tau^{+} \tau^{-}$have already been measured with high precision at the LHC. From these signal strengths, one can obtain information on the couplings of the Higgs boson to SM particles and derive constraints on the extension models. For the model considered in this work and in the narrow width approximation, the signal strength of $\mathcal{H}$ is [28]

$$
\mu_{\mathcal{H}}=\frac{\Gamma_{\mathcal{H}}^{\mathrm{SM}} \cos ^{4} \theta}{\Gamma_{\mathcal{H}}^{\mathrm{SM}} \cos ^{2} \theta+\Gamma_{\mathcal{H} \rightarrow \chi \chi}+\Gamma_{\mathcal{H} \rightarrow \mathcal{S S}}},
$$

where $\Gamma_{\mathcal{H}}^{\mathrm{SM}}$ denotes the total decay widths of the SM-like Higgs boson with mass being set to $m_{\mathcal{H}}$, and $\Gamma_{\mathcal{H} \rightarrow X X}$ is the width of $\mathcal{H}$ decaying to a pair of $X(=\chi, \mathcal{H})$, which can be found in appendix D. We see that the signal strengths of $\mathcal{H}$ is suppressed by two factors: $\cos ^{2} \theta$ and the presence of new decay channels. Even when the new decay channels are kinematically forbidden, the signal strength is still reduced by $\cos ^{2} \theta$. This means that a generic signature of the mixing of the SM Higgs boson with an extra singlet scalar boson can be derived from a reduced signal of the Higgs bosons at the LHC [28].

We use the HiggsSignals_v1.4.0 package [97] to estimate the $\chi^{2}$ of a given model and assess which sample points are allowed by the signal strength measurements. The package assumes a Gaussian probability distribution and uses the peak-centered method for the calculation of $\chi^{2}=\chi_{\mu}^{2}+\chi_{m}^{2}$, where $\chi_{\mu}^{2}$ is evaluated by comparing the signal strength measurements for the peak to the model-predicted signal strengths and $\chi_{m}^{2}$ is evaluated by comparing the model-predicted Higgs boson mass and the observed one if a mass measurement is also available [97]. The signal strength measurements used in the HiggsSignals analysis are summarized in table 1.

We find that $\chi_{\min }^{2}$, the minimum of $\chi^{2}$, is obtained in the model with the mixing angle $\theta=0$, which means no mixing for the SM Higgs boson. Totally 89 observables are used in each fit, and $\chi_{\min }^{2}=102.02$. We conclude that a sample point in the parameter space is 


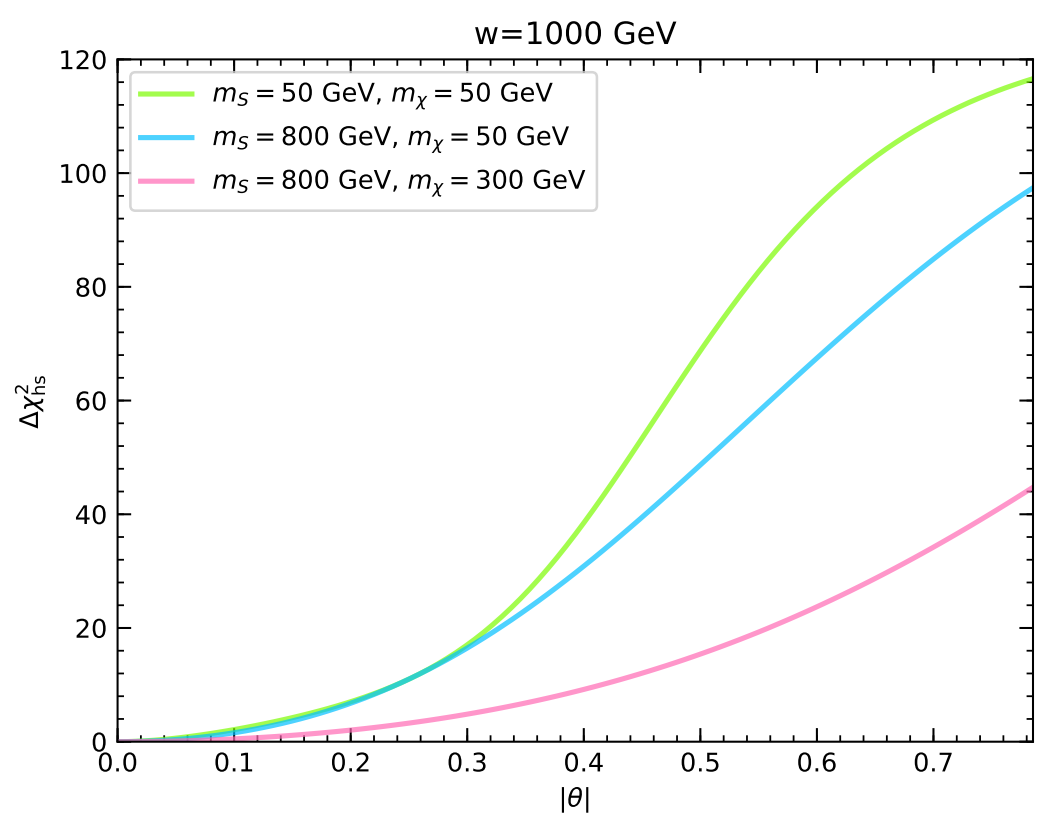

Figure 7. $\Delta \chi_{\text {hs }}^{2}$ as a function of $|\theta|$, with $w=1000 \mathrm{GeV}$. The green, blue, and pink curves represent the results with $\left(m_{\mathcal{S}}, m_{\chi}\right)=(50,50) \mathrm{GeV},(800,50) \mathrm{GeV}$, and $(800,300) \mathrm{GeV}$ respectively.

not excluded by the experimental results at $95 \%$ CL if $\Delta \chi_{\mathrm{hs}}^{2}=\chi^{2}-\chi_{\min }^{2}<9.49$ (for four free parameters).

We plot $\Delta \chi_{\text {hs }}^{2}$ as a function of the mixing angle in figure 7 , with the pink, blue, and green curves denoting the results for $\left(m_{\mathcal{S}}, m_{\chi}\right)=(800,300) \mathrm{GeV},(800,50) \mathrm{GeV}$, and $(50,50) \mathrm{GeV}$, respectively. As discussed above, when $m_{\mathcal{S}}, m_{\chi}>m_{\mathcal{H}} / 2$, the SM Higgs signal strength is reduced by a factor of $\cos ^{2} \theta$, and thus $\Delta \chi_{\text {hs }}^{2}$ increases with $|\theta|$. When $m_{\chi}<m_{\mathcal{H}} / 2$, the SM Higgs invisible decay $\mathcal{H} \rightarrow \chi \chi$ is kinematically allowed, leading to an additional suppression in the SM Higgs signal strength, as is indicated by the blue curve. One can also see that the opening of new decay channels will play a dominant role in suppressing the SM Higgs signal strength. Furthermore, if $m_{\mathcal{S}}<m_{\mathcal{H}} / 2$ the decay channel $\mathcal{H} \rightarrow \mathcal{S S}$ opens up and dominates $\Delta \chi_{\mathrm{hs}}^{2}$ for $|\theta| \gtrsim 0.3$, as shown by the green curve.

In summary, the constraints from Higgs signal strength measurements can be divided into two cases:

- Case 1. If all of the extra particles are heavier than half of the SM Higgs boson mass, the Higgs signal strength scales as $\cos ^{2} \theta$. The mixing angle of the SM Higgs boson with an extra singlet scalar should be $\lesssim 0.4\left(23^{\circ}\right)$ at $95 \%$ CL.

- Case 2. If there is at least one extra particle is lighter than half of the SM Higgs boson mass, the Higgs signal strength will receive an additional suppression, the constraint on the mixing angle becomes more rigorous than Case 1. 


\begin{tabular}{|l|c|c|c|}
\hline \multirow{2}{*}{ Channel } & \multicolumn{2}{|c|}{ Signal strength } & \multirow{2}{*}{ refs. } \\
\cline { 2 - 3 } & ATLAS & CMS & \\
\hline$h \rightarrow \gamma \gamma$ & $1.17_{-0.26}^{+0.28}$ & $1.14_{-0.23}^{+0.26}$ & {$[98,99]$} \\
$h \rightarrow W W^{*}$ & $1.18_{-0.21}^{+0.24}$ & $0.72_{-0.18}^{+0.20}$ & {$[98,100]$} \\
$h \rightarrow Z Z^{*}$ & $1.46_{-0.34}^{+0.40}$ & $0.93_{-0.25}^{+0.29}$ & {$[98,101]$} \\
$h \rightarrow b \bar{b}$ & $0.63_{-0.37}^{+0.39}$ & $1.00_{-0.50}^{+0.50}$ & {$[98,102]$} \\
$h \rightarrow \tau^{+} \tau^{-}$ & $1.44_{-0.37}^{+0.42}$ & $0.78_{-0.27}^{+0.27}$ & {$[98,102]$} \\
\hline
\end{tabular}

Table 1. Experimental results of Higgs signal strength measurements in various channels that are used in the HiggsSignals analysis [97].

\subsection{Results}

We show our results in figure 8 after combining all the collider experimental constraints discussed above. We find that 8104 (in the green histogram) out of 18047 sample points (in the blue histogram) that can trigger a sufficiently strong EWPT survive the experimental bounds. As discussed above, the most stringent constraints come from the Higgs signal strength measurements.

As shown by the blue histogram, all of the $m_{\mathcal{S}}$ and most of the $m_{\chi}$ sample points that induce a strong EWPT distribute at masses larger than $m_{\mathcal{H}} / 2=62.5 \mathrm{GeV}$. According to the above discussions, we see that there is a universal constraint on the mixing angle, $|\theta| \lesssim 0.4$, from the Higgs signal strength measurements. Here we have chosen the scan range of $m_{\mathcal{S}} \geq 150 \mathrm{GeV}$, apparently, the bound on the mixing angle can be much stronger if $m_{\mathcal{S}}$ and/or $m_{\chi}$ is lighter than $m_{\mathcal{H}} / 2$. Anyway, the constraint $|\theta| \lesssim 0.4$ is not dependent on the assumed parameter ranges. Hence, as shown by the lower right plot of figure 8 , there is a hard cut-off around \pm 0.4 in the distribution of $\theta$.

The peak around $200-400 \mathrm{GeV}$ in the distribution of $m_{\chi}$ is now removed, and it tends to a flater distribution in the range $100-1500 \mathrm{GeV}$. While there seems to be no preferred range in the $w$ distribution by the signal strength constraints, the sample points with $m_{\mathcal{S}} \lesssim 1.2 \mathrm{TeV}$ are strongly disfavored, as shown in the top two plots. For one thing, the signal strength bounds require a mixing angle $|\theta| \lesssim 0.4$. Yet samples with large values of mixing angle are mainly associated with a lighter scalar mass $m_{\mathcal{S}}$ due to the perturbative unitarity. For another reason, the scalar mass $m_{\mathcal{S}}$ should be large enough to induce a strong EWPT when the mixing angle is small.

We summarize our main conclusions obtained in this section below:

- The Higgs signal strength measurements give a universal constraint on the mixing angle $|\theta| \lesssim 0.4\left(23^{\circ}\right)$.

- The mass of heavy scalar $\mathcal{S}$ should be larger than $1.2 \mathrm{TeV}$ from the combined constraints of Higgs signal strength measurements and perturbative unitarity and the requirement of strong EWPT. 

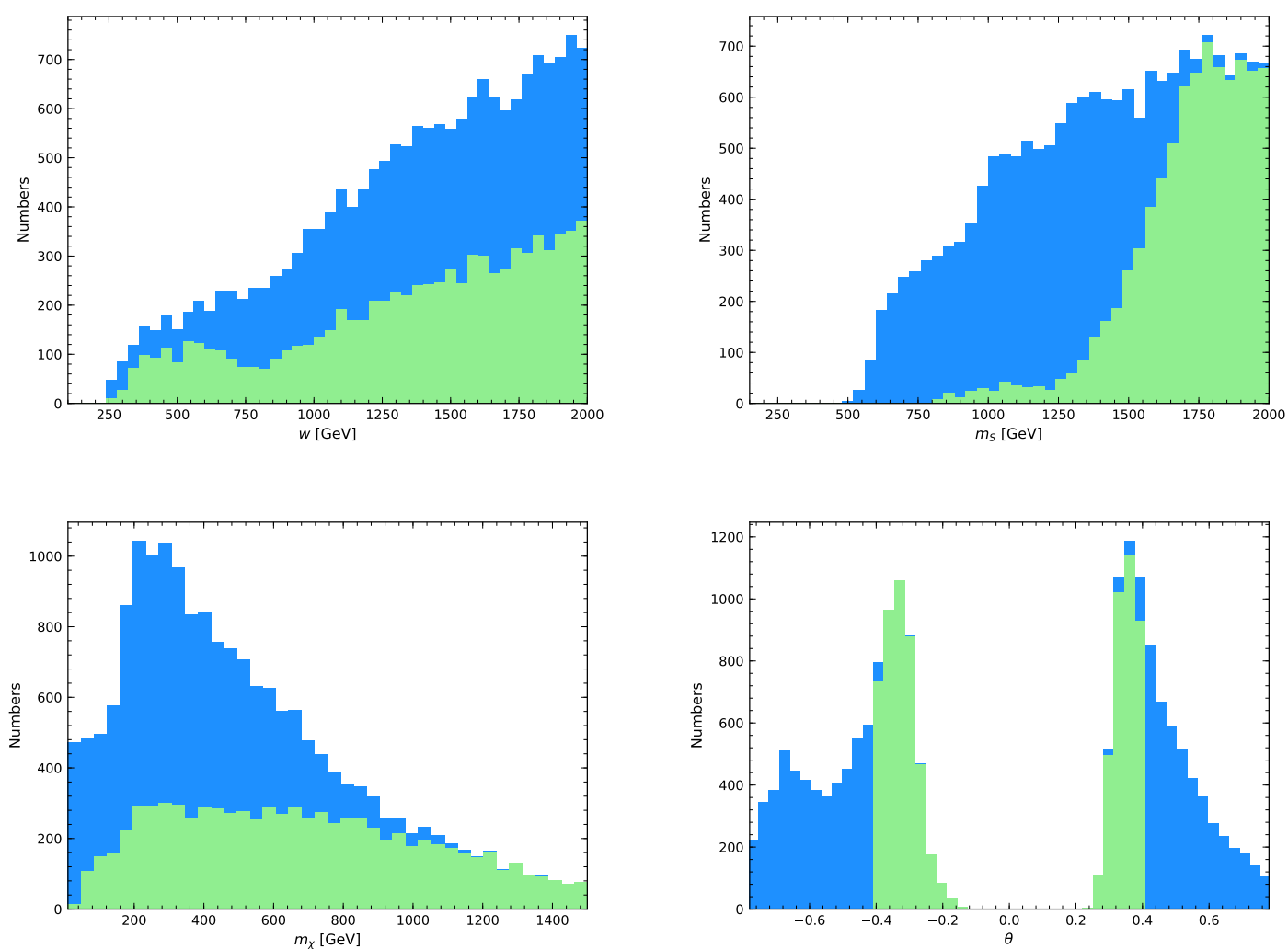

Figure 8. Distributions of the input parameters. The blue histogram represents those samples that can trigger a strong EWPT, the green histogram represents those samples that further survive the experimental constraints.

- Our analysis supports $|\theta| \gtrsim 0.2\left(11.5^{\circ}\right)$ for the scalar mass $m_{\mathcal{S}} \lesssim 2 \mathrm{TeV}$, which is the requirement of a sufficiently strong EWPT. However, this conclusion depends on the scanning range of scalar mass $m_{\mathcal{S}}$ pre-assumed in this study. A strong EWPT for the mixing angle less than 0.2 might be available if the scanned heavy scalar mass extends beyond $2 \mathrm{TeV}$ (we leave this for future studies).

\section{Dark matter phenomenology}

In this section, we discuss constraints on the model from the observed DM relic density and null direct search result.

\subsection{Dark matter relic density}

As mentioned above, the pseudo-Goldstone boson $\chi$ from the spontaneous symmetry breaking has a $\mathbb{Z}_{2}$ symmetry, ensuring the stability of the pseudo-Goldstone boson as a DM candidate. In the standard freeze-out scenario, the DM particles are in chemical equilibrium with the other SM particles via annihilation-production reaction in the early Universe. The DM population becomes nonrelativistic and the annihilations take over the thermal 

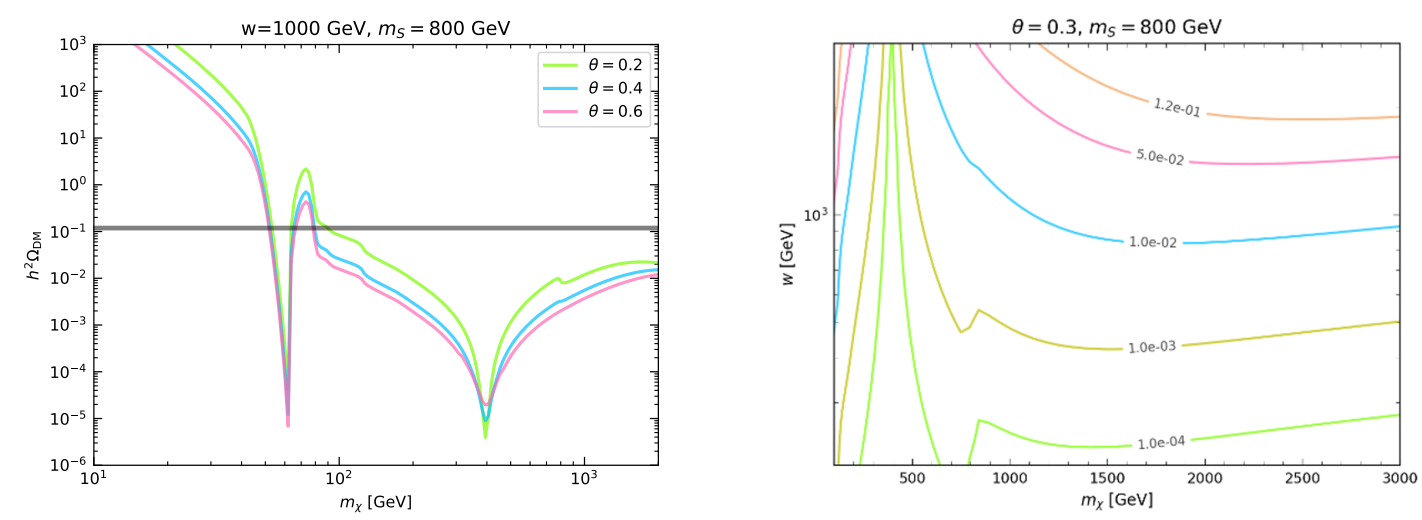

Figure 9. Left plot: DM thermal relic density as a function of the DM mass for $w=1000 \mathrm{GeV}$, $m_{\mathcal{S}}=800 \mathrm{GeV}$, and $\theta=0.2$ (green), 0.4 (light blue) and 0.6 (green). The black line denotes the DM relic density given by the Planck satellite's observation of the CMB radiation [104] Right plot: contours of the DM thermal relic density in the $m_{\chi}-w$ plane for $\theta=0.3$ and $m_{\mathcal{S}}=800 \mathrm{GeV}$.

productions with the adiabatic expansion of the Universe. At around the temperature that the reaction rate falls below the Universe expansion rate, the DM particles begin to decouple from the thermal bath. The evolution of the DM number density is described by the Boltzmann equation

$$
\frac{d Y}{d T}=\sqrt{\frac{\pi g_{*}(T)}{45}} M_{\mathrm{pl}}\left\langle\sigma v_{\mathrm{rel}}\right\rangle\left[Y(T)^{2}-Y_{\mathrm{eq}}(T)^{2}\right],
$$

where the abundance $Y(T)$ denotes the ratio of the DM number density $n_{\chi}$ to the entropy density, $M_{\mathrm{pl}}=1.22 \times 10^{19} \mathrm{GeV}$ is the Planck mass, $g_{*}$ is the effective number of relativistic degrees of freedom, $Y_{\text {eq }}(T)$ is the thermal equilibrium abundance, and $\left\langle\sigma v_{\text {rel }}\right\rangle$ is the relativistic thermally averaged annihilation cross section. The resulting DM relic density is given by

$$
h^{2} \Omega_{\mathrm{DM}}=2.742 \times 10^{8} Y_{0} \frac{m_{\chi}}{\mathrm{GeV}},
$$

where $Y_{0}$ is the abundance of DM in the present Universe. In our numerical analysis, we make use of the MicrOMEGAs_5.0.4 package [103] to calculate the DM relic density.

In the left plot of figure 9 , we plot the DM relic density $h^{2} \Omega_{\mathrm{DM}}$ as a function of the DM mass $m_{\chi}$, with the values of $w$ and $m_{\mathcal{S}}$ fixed at $1000 \mathrm{GeV}$ and $800 \mathrm{GeV}$, respectively. In our model, the DM annihilation to the SM particles are mediated by the SM-like Higgs boson $\mathcal{H}$ and heavy scalar $\mathcal{S}$. When $m_{\chi} \lesssim m_{\mathcal{H}} / 2$, its annihilation process is kinematically suppressed, leading to a large value of DM relic density. The resonant DM annihilation occurs at $m_{\chi} \simeq m_{\mathcal{H}} / 2$ and $m_{\mathcal{S}} / 2$, which would result in a sharp decrease of the DM relic density, as shown by the two dips in the curves. In the right plot of figure 9 , we plot the contours of DM relic density in the $m_{\mathcal{S}^{-}} w$ plane. We see that the DM relic density becomes larger as $w$ increases.

In the left plot of figure 10, we calculate the DM relic density of the sample points. The blue scatter points represent the samples that have a sufficiently strong EWPT and 

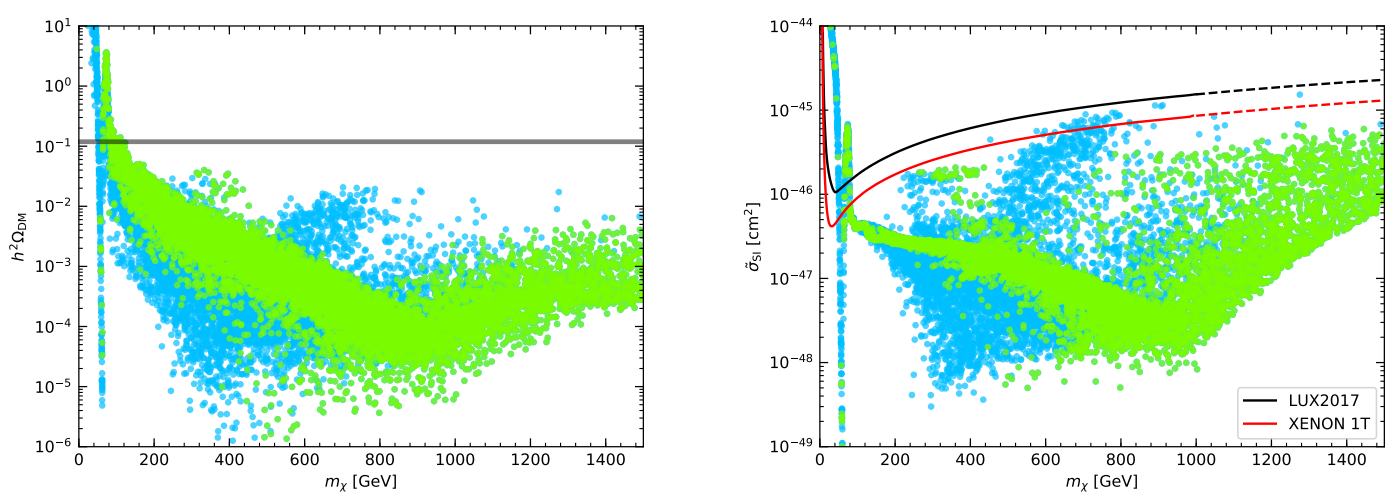

Figure 10. Left plot: DM thermal relic density as a function of the DM mass. Right plot: effective spin-independent DM-nucleon elastic scattering cross section as a function of the DM mass. The black and red curves denote the upper limits on scattering cross section from LUX [52] and XENON1T [54] experiments, respectively. In both plots, the blue scatter points represent the samples that have a sufficiently strong EWPT and the green scatter points are those further surviving the experimental constraints.

the green scatter points are the samples further survive all the experimental constraints. The black line denotes the DM relic density given by the Planck satellite's observation of the CMB radiation [104]

$$
h^{2} \Omega_{\mathrm{DM}}^{\mathrm{obs}}=0.1188 \pm 0.0010 .
$$

It is seen that most of the samples have $h^{2} \Omega_{\mathrm{DM}}$ much below the observed result. On one hand, these sample parameters can evade from a over-closed Universe. On the other hand, only a small fraction of DM consists of the pseudo-Goldstone boson from spontaneous symmetry breaking in our model.

\subsection{Direct detection}

For DM direct detection, we use the MicrOMEGAs package to compute the spin-independent DM-nucleon elastic scattering cross section $\sigma_{\mathrm{SI}}$. As shown above, for the parameter space considered here, only a small fraction of DM is made up of the pseudo-Goldstone bosons. To compare with the experimental upper bounds, we have to scale the scattering cross section as

$$
\tilde{\sigma}_{\mathrm{SI}}=f_{X} \sigma_{\mathrm{SI}}, \quad \text { where } f_{X}=\frac{h^{2} \Omega_{\mathrm{DM}}}{0.1188} .
$$

We simultaneously calculate the DM relic density $h^{2} \Omega_{\mathrm{DM}}$ and scattering cross section $\sigma_{\mathrm{SI}}$ with the help of MicrOMEGAs, then we obtain the effective scattering cross section $\tilde{\sigma}_{\text {SI }}$ using eq. (7.4). We plot our results in the right plot of figure 10. Again, the blue scatter points represent the samples that have a sufficiently strong EWPT, and the green scatter points are the samples further surviving all the collider constraints. The black curve denotes the upper limit on DM-nucleon elastic scattering cross section from the LUX [52] experiment, and the red curve from the XENON1T [54] experiment. They are the most stringent constraints on the spin-independent DM-nucleon scattering cross section up to date. The 
scattering cross section in our model is suppressed by both a small mixing angle $\theta$ and a large value of $w$. It can be seen that our scanned sample points have sustained the most stringent constraints from DM direct detection experiments with most of the scattering cross sections being much below the upper limits from the direct detections.

\section{Gravitational waves}

A first-order cosmological phase transition can only occur in the presence of a scalar effective potential barrier separating the symmetry-broken and -unbroken vacua. Although the probability of tunneling from the metastable minimum to the stable one via the instantons is very tiny, the decay of the false vacuum can proceed through thermal fluctuations which help overcome the potential barrier. The tunneling rate per unit volume and time element is approximately given by $[49,105]$

$$
\Gamma(T)=A(T) e^{-S_{3} / T},
$$

where $A(T) \simeq\left[S_{3} /(2 \pi T)\right]^{3 / 2} T^{4}$ and $S_{3}$ denotes the three-dimensional on-shell Euclidean action of a instanton. Due to the supercooling effect, the onset of the bubble nucleations can be delayed to a temperature $T_{\mathrm{n}}$ smaller than the critical temperature $T_{\mathrm{c}}$. The nucleation temperature $T_{\mathrm{n}}$ is defined to be at which the probability of nucleating one bubble per horizon volume is of order one, i.e., $p(T) \sim 1$, where the probability of bubble nucleations per Hubble volume is defined as

$$
p(T)=\int_{T}^{T_{\mathrm{c}}} \frac{\Gamma(x)}{H^{4}(x)} \frac{d x}{x} \approx\left(\frac{T}{H}\right)^{4} e^{-S_{3} / T} .
$$

In a radiation dominated Universe, the Hubble parameter is given by $H^{2}=8 \pi^{3} g_{*} T^{4} /\left(90 M_{\mathrm{pl}}^{3}\right)$ and $g_{*} \simeq 110$. The condition $p(T) \sim 1$ guarantees the percolation of bubbles in the early Universe and can be translated into the following criterion for determining the nucleation temperature [49]

$$
\frac{S_{3}\left(T_{\mathrm{n}}\right)}{T_{\mathrm{n}}} \simeq 4 \ln \left(\frac{T_{\mathrm{n}}}{H}\right) \simeq 142-4 \log \left(\frac{T_{\mathrm{n}}}{246 \mathrm{GeV}}\right)
$$

For the phase transition at a characteristic temperature $T \sim \mathcal{O}(100 \mathrm{GeV})$, the condition above is well approximated by $S_{3}\left(T_{\mathrm{n}}\right) / T_{\mathrm{n}} \simeq 140$. The successful bubble nucleations at EW scale are guaranteed by eq. (8.3), which requires a sufficiently large bubble nucleation rate to overcome the expansion rate. On one hand, a sufficiently strong EWPT ensures that the washout of baryon asymmetry through sphalerons is suppressed. On the other hand, a successful bubble nucleation is the requirement of triggering baryogenesis in the EW broken phase. The latter is typically a more stringent requirement on the model.

The Euclidean action for a spherical bubble configuration can be written as

$$
S_{3}(T)=4 \pi \int d r r^{2}\left[\frac{1}{2}\left(\frac{d \Phi}{d r}\right)^{2}+V_{\mathrm{eff}}(\Phi, T)\right] .
$$


By extremizing the Euclidean action, we obtain the following differential equation

$$
\frac{d^{2} \Phi}{d r^{2}}+\frac{2}{r} \frac{d \Phi}{d r}-\frac{d V}{d \Phi}=0
$$

with the boundary conditions

$$
\left.\frac{d \Phi}{d r}\right|_{r=0}=0,\left.\Phi\right|_{r \rightarrow \infty}=0 .
$$

If $\Phi(r)$ represents the profile of a particle in the potential $V$, then eq. (8.5) can be treated as the classical equation of motion, which can be solved by the traditional overshooting/undershooting method [105]. In this work, we employ the CosmoTransitions 2.0.2 package [106] to perform the numerical calculations of the bubble profile and Euclidean action. Afterwards, we use eq. (8.3) to determine the nucleation temperature $T_{n}$.

\subsection{Gravitational wave parameters}

It has been shown that the stochastic gravitational waves (GW's) produced from a cosmological phase transition can be fully characterized by the knowledge of two primary parameters [107], which are defined as

$$
\alpha=\frac{\epsilon\left(T_{*}\right)}{\rho_{\mathrm{rad}}\left(T_{*}\right)} \text { and } \frac{\beta}{H_{*}}=\left.T_{*} \frac{d}{d T}\left(\frac{S_{3}(T)}{T}\right)\right|_{T=T_{*}},
$$

where $T_{*}$ is the GW generation temperature, $\rho_{\text {rad }}=\pi^{2} g_{*} T^{4} / 30$ is the radiation energy density in the plasma, and the latent heat associated with the phase transition is given by

$$
\epsilon(T)=T \frac{\partial \Delta V_{b s}(T)}{\partial T}-\Delta V_{b s}(T),
$$

where $\Delta V_{b s}(T) \equiv V_{\text {eff }}(v(T), w(T), T)-V_{\text {eff }}\left(0, w_{0}(T), T\right)$ is the potential difference between the broken phase and the symmetric phase at temperature $T$. Therefore, the parameter $\alpha$ is related to the maximum available energy budget for gravitational wave emissions. The parameter $\beta$ represents the rate of time variation of the nucleation rate, whose inverse gives the duration of the bubble nucleation. Consequently, $\beta / H_{*}$ defines the characteristic frequency of the GW spectrum produced from the phase transition.

In addition to the GW parameters $\alpha$ and $\beta / H_{*}$ and the nucleation temperature $T_{n}$, the GW spectrum also depends on the bubble wall velocity $v_{w}$, which is the expanding speed of the true vacuum. It has been pointed out in refs. [108, 109] that it is the relative velocity between the wall and the plasma in the front $\left(v_{+}\right)$rather than $v_{w}$ that should be used in the calculations of EW baryogenesis. For a strong EWPT, the condition $v_{+} \ll v_{w}$ could be achieved, making it possible to generate a viable EW baryogenesis and a loud GW signal in the same scenario. In this work, the bubble velocity $v_{w}$ is simply assigned to be around 1 for the calculations of GW spectra.

We show the calculation results of parameters $\alpha$ and $\beta / H_{*}$ in the left plot of figure 11, the colored bar indicates the nucleation temperature $T_{n}$. The distribution of $T_{n}$ are given in the right plot of figure 11 . We find that 8564 out of 18047 sample points satisfy the bub- 

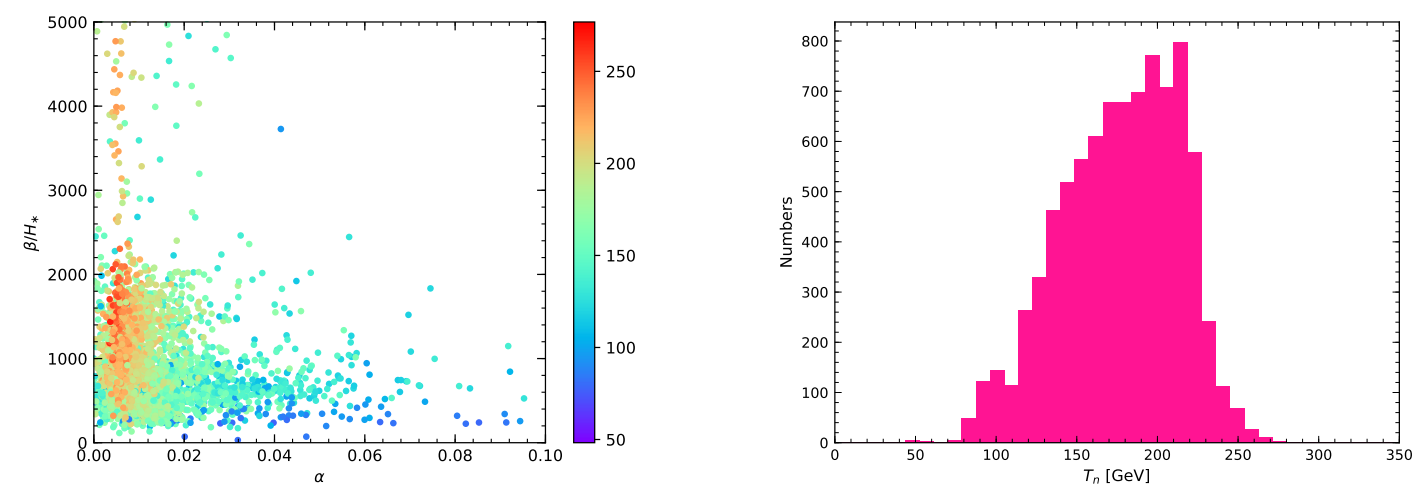

Figure 11. Left plot: distributions of the GW parameters $\alpha$ and $\beta / H_{*}$. The colored bar indicates the nucleation temperature $T_{n}$. Right plot: distribution of nucleation temperature $T_{n}$.

ble nucleation condition in eq. (8.3). Note that in the calculations of $\beta / H_{*}$ with eq. (8.7), we have assumed that reheating is not significant for typical transitions. In this case, the temperature for $\mathrm{GW}$ generation $T_{*}$ is approximately equivalent to the nucleation temperature $T_{n}$, i.e., $T_{*} \simeq T_{n}$. A strong supercooling could not only enhance the strength of the phase transition, but also change the evolution of the Universe since the expansion of the Universe would be dominated by vacuum energy in the supercooled phase, instead of radiation. In this case, there is a lower bound on the temperature of the transition to ensure the successful bubble percolation and completion of the EWPT [110].

\subsection{Gravitational wave spectrum}

In what follows we review the three processes that are involved in the production of GW's during a first-order phase transition (see refs. [111, 112] and references therein for details):

- Collisions of bubble walls and shocks in the plasma. GW's produced from this process depends only on the dynamics of the scalar field. The "envelope approximation" is used in the numerical simulations to estimate the GW spectrum, given by [113] (analytical calculations of the GW spectrum from this process can be found in ref. [114])

$$
h^{2} \Omega_{\mathrm{col}}(f)=1.67 \times 10^{-5}\left(\frac{H_{*}}{\beta}\right)^{2}\left(\frac{\kappa_{\mathrm{col} \alpha}}{1+\alpha}\right)^{2}\left(\frac{100}{g_{*}}\right)^{\frac{1}{3}}\left(\frac{0.11 v_{w}^{3}}{0.42+v_{w}^{2}}\right) S_{\mathrm{col}}(f) .
$$

- Sound waves in the plasma generated subsequently after the bubble collisions. $\mathrm{Nu}-$ merical simulations indicate that the durations of sound waves and turbulence as active sources of GW's are typically much longer than the collisions of the bubble walls. This process contributes a GW spectrum desribed by [115]

$$
h^{2} \Omega_{\mathrm{sw}}(f)=2.65 \times 10^{-6}\left(\frac{H_{*}}{\beta}\right)\left(\frac{\kappa_{\mathrm{sw}} \alpha}{1+\alpha}\right)^{2}\left(\frac{100}{g_{*}}\right)^{\frac{1}{3}} v_{w} S_{\mathrm{sw}}(f) .
$$

- Turbulence in the plasma formation after the bubble collisions. Simulations show that only a small fraction $\epsilon \sim 5-10 \%$ of the bulk motion from the bubble walls is 
converted into turbulence. However, GW's produced from this process could play a dominant role at high frequencies, as the GW signals from sound waves decay much faster. The GW spectrum from turbulence can be parameterized as [116]

$$
h^{2} \Omega_{\text {turb }}(f)=3.35 \times 10^{-4}\left(\frac{H_{*}}{\beta}\right)\left(\frac{\kappa_{\text {turb }} \alpha}{1+\alpha}\right)^{\frac{3}{2}}\left(\frac{100}{g_{*}}\right)^{1 / 3} v_{w} S_{\text {turb }}(f) .
$$

The efficiency factors $\kappa_{\mathrm{col}}, \kappa_{\mathrm{sw}}$, and $\kappa_{\mathrm{turb}}$ indicate respectively the fractions of latent heat that are transformed into the kinetic energy of bubbles, the bulk motion of the plasma, and the turbulence and finally into GW's. Thus they are functions of $\alpha$. The total stochastic GW spectrum is approximately given by adding up these three contributions:

$$
h^{2} \Omega_{\mathrm{GW}} \simeq h^{2} \Omega_{\mathrm{col}}+h^{2} \Omega_{\mathrm{sw}}+h^{2} \Omega_{\mathrm{turb}}
$$

The spectral shapes in eqs. (8.9)-(8.11) are given by

$$
\begin{aligned}
S_{\mathrm{col}}(f) & =\frac{3.8\left(f / f_{\mathrm{col}}\right)^{2.8}}{1+2.8\left(f / f_{\mathrm{col}}\right)^{3.8}}, \\
S_{\mathrm{sw}}(f) & =\left(f / f_{\mathrm{sw}}\right)^{3}\left(\frac{7}{4+3\left(f / f_{\mathrm{sw}}\right)^{2}}\right)^{7 / 2}, \\
S_{\text {turb }}(f) & =\frac{\left(f / f_{\text {turb }}\right)^{3}}{\left[1+\left(f / f_{\text {turb }}\right]^{\frac{11}{3}}\left(1+8 \pi f / H_{0}\right)\right.},
\end{aligned}
$$

where the red-shifted Hubble constant observed today is given by

$$
H_{0}=16.5 \times 10^{-3} \mathrm{mHz}\left(\frac{T_{*}}{100 \mathrm{GeV}}\right)\left(\frac{g_{*}}{100}\right)^{\frac{1}{6}} .
$$

The frequency $f_{*}$ with respect to the Hubble scale at the nucleation temperature $T_{*}$ is red-shifted to the frequency $f$ today by $f_{*} / H_{*}=f / H_{0}$. We write out the red-shifted peak frequency today as follows:

$$
\begin{aligned}
f_{\mathrm{col}} & =16.5 \times 10^{-3} \mathrm{mHz}\left(\frac{0.62}{1.8-0.1 v_{w}+v_{w}^{2}}\right)\left(\frac{\beta}{H_{*}}\right)\left(\frac{T_{*}}{100 \mathrm{GeV}}\right)\left(\frac{g_{*}}{100}\right)^{\frac{1}{6}}, \\
f_{\mathrm{sw}} & =1.9 \times 10^{-2} \mathrm{mHz} \frac{1}{v_{w}}\left(\frac{\beta}{H_{*}}\right)\left(\frac{T_{*}}{100 \mathrm{GeV}}\right)\left(\frac{g_{*}}{100}\right)^{\frac{1}{6}} \\
f_{\text {turb }} & =2.7 \times 10^{-2} \mathrm{mHz} \frac{1}{v_{w}}\left(\frac{\beta}{H_{*}}\right)\left(\frac{T_{*}}{100 \mathrm{GeV}}\right)\left(\frac{g_{*}}{100}\right)^{\frac{1}{6}} .
\end{aligned}
$$

There is a critical phase transition strength $\alpha_{\infty}$ for the phase transition, which is estimated as

$$
\alpha_{\infty} \simeq \frac{30}{24 \pi^{2}} \frac{\sum_{i} n_{i} \Delta M_{i}^{2}\left(\Phi_{*}\right)}{g_{*} T_{*}^{2}}
$$

where $n_{i}$ is equal to $N_{i}$ for bosons and $N_{i} / 2$ for fermions, with $N_{i}$ already given in section 3. $\Delta M_{i}^{2}\left(\Phi_{*}\right)$ is the difference of the field-dependent squared masses between the symmetric and broken phases. According to $\alpha_{\infty}$, the phase transition can be divided into two cases [111]: 
- Case 1. Non-runaway bubbles, $\alpha<\alpha_{\infty}$. In this case, the bubble walls will reach a terminal velocity and the latent energy transferred into the scalar field is negligible, i.e., $\kappa_{\text {col }} \simeq 0$. The efficiency factor for the sound wave contribution is then given by

$$
\kappa_{\mathrm{sw}} \simeq \frac{\alpha}{0.73+0.083 \sqrt{\alpha}+\alpha}, \text { for } v_{w} \sim 1 .
$$

The efficiency factor for turbulence $\kappa_{\mathrm{turb}}$ is related to $\kappa_{\mathrm{sw}}$ by $\kappa_{\mathrm{turb}}=\epsilon \kappa_{\mathrm{sw}}$, where we take $\epsilon=0.1$ in this work.

- Case 2. Runaway bubbles, $\alpha>\alpha_{\infty}$. In this case, the bubble walls can accelerate continuously and finally run away. The fraction $\kappa_{\text {col }}=1-\alpha_{\infty} / \alpha$ of the total latent energy goes into accelerating the bubble wall, and the other fraction $\alpha_{\infty} / \alpha$ of the latent energy is transformed into bulk motion and thermal energy. The efficiency factor $\kappa_{\mathrm{sw}}=\kappa\left(\alpha_{\infty}\right) \alpha_{\infty} / \alpha$, where $\kappa\left(\alpha_{\infty}\right)$ is calculated using eq. (8.17).

A recent study [117] suggests that although bubbles may runaway in certain cases, most of their energy is dissipated into the surrounding plasma and very little energy is deposited in the bubble walls. This in turn leads to a negligible contribution to the GW spectrum from bubble collisions. Hence, we only take the GW spectra produced by sound waves and turbulence into account in our calculations.

\subsection{Space-based interferometers}

The frequentist approach is normally used for the experimental investigation of the stochastic GW signals from EWPT, where the detectability of the signals is measured by the corresponding signal-to-noise ratio (SNR) $[37,111]$

$$
\rho=\sqrt{\mathcal{N} \mathcal{T}_{\text {obs }} \int_{f_{\text {min }}}^{f_{\max }} d f\left[\frac{h^{2} \Omega_{\mathrm{GW}}(f)}{h^{2} \Omega_{\exp }(f)}\right]^{2}},
$$

where $h^{2} \Omega_{\exp }$ denotes the sensitivity of a GW experiment, $\mathcal{N}$ is the number of independent observatories of the experiment, and $\mathcal{T}_{\text {obs }}$ is the duration of the mission in units of year.

The peak frequency of GW spectrum produced from the EWPT is red-shifted to around the milli-Hertz band, which falls right within the range of future space-based GW interferometers. The planned space-based GW experiments considered in this work include the LISA [62] interferometer as well as the proposed successors B-DECIGO [118], DECIGO [63], and BBO [64]. For the auto-correlated experiments LISA and B-DECIGO, $\mathcal{N}=1$, while $\mathcal{N}=2$ for the cross-correlated experiments DECIGO and BBO. Following ref. [37], we assume a mission duration of $\mathcal{T}_{\text {obs }}=4$ years for all of the experiments. The SNR threshold value $\rho_{\mathrm{thr}}$, above which the GW signal is detectable for the experiment, and the other detector parameters are summarized in table 2. The experimental sensitivities are summarized in appendix E.

In figure 12 , the GW experimental sensitivities are shown in the $\alpha-\beta / H_{*}$ plane by the colored regions, in which the SNR of a given GW observatory exceeds its detection threshold. We have assumed a nucleation temperature $T_{n}=200 \mathrm{GeV}$ for the determination 


\begin{tabular}{|l|l|c|c|c|c|}
\hline Experiment & Frequency range & $\rho_{\text {thr }}$ & $\mathcal{N}$ & $\mathcal{T}_{\text {obs }}[\mathrm{yrs}]$ & Refs. \\
\hline LISA & $10^{-5}-1 \mathrm{~Hz}$ & 10 & 1 & 4 & {$[62,119]$} \\
B-DECIGO & $10^{-2}-10^{2} \mathrm{~Hz}$ & 8 & 1 & 4 & {$[118,120]$} \\
DECIGO & $10^{-3}-10^{2} \mathrm{~Hz}$ & 10 & 2 & 4 & {$[63,121,122]$} \\
BBO & $10^{-3}-10^{2} \mathrm{~Hz}$ & 10 & 2 & 4 & {$[64,121,122]$} \\
\hline
\end{tabular}

Table 2. A summary of parameters and assumptions used for the planned space-based interferometers.
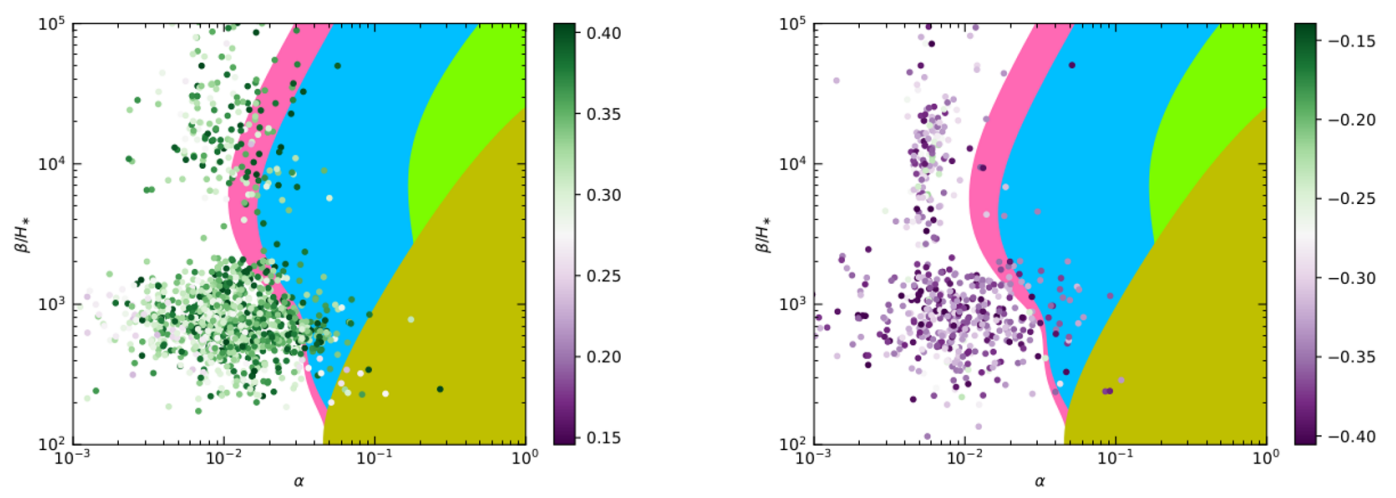

Figure 12. The pink, blue, yellow, and green regions represent respectively the sensitivities of BBO, DECIGO, LISA, and B-DECIGO exceeding the detection threshold, assuming the nucleation temperature $T_{n}=200 \mathrm{GeV}$. The scatter points are the samples that both generate a strong firstorder EWPT and survive the collider searches and DM experiments. The colored bar shows the values of $\theta$. The samples with $\theta>0$ are plotted in the left plot, while the samples with $\theta<0$ are plotted in the right plot. 11

of these regions. The scatter points are the samples that both generate a strong first-order EWPT and survive the collider searches and DM experiments. The colored bar shows values of the mixing angle $\theta$. The samples with $\theta>0(\theta<0)$ are plotted in the left (right) plot of figure 12). As illustrated by this figure, a considerable portion of sample points with a mixing angle $|\theta|$ in the range $0.25-0.4$ could be detected by BBO and DECIGO. A few samples have extended into the region in which the GW signal is large enough to be detected by the LISA experiment. We thus expect that the EWPT scenario depicted in this work will be tested by the future space-based interferometers.

\section{Gauge dependence}

In this section, we scrutinize the issue of gauge dependence in our conclusions drawn above. It has been pointed out that the SM one-loop effective potential depends on the gauge parameter $\xi$ in the $R_{\xi}$ gauge due to the thermal corrections to the masses. Ref. [80] proposed a method to determine the gauge-independent $v_{c}$ and $v_{c} / T_{c}$ in the high-temperature approximation. With this method, a gauge-invariant, perturbative computation of $v_{c}$ and 

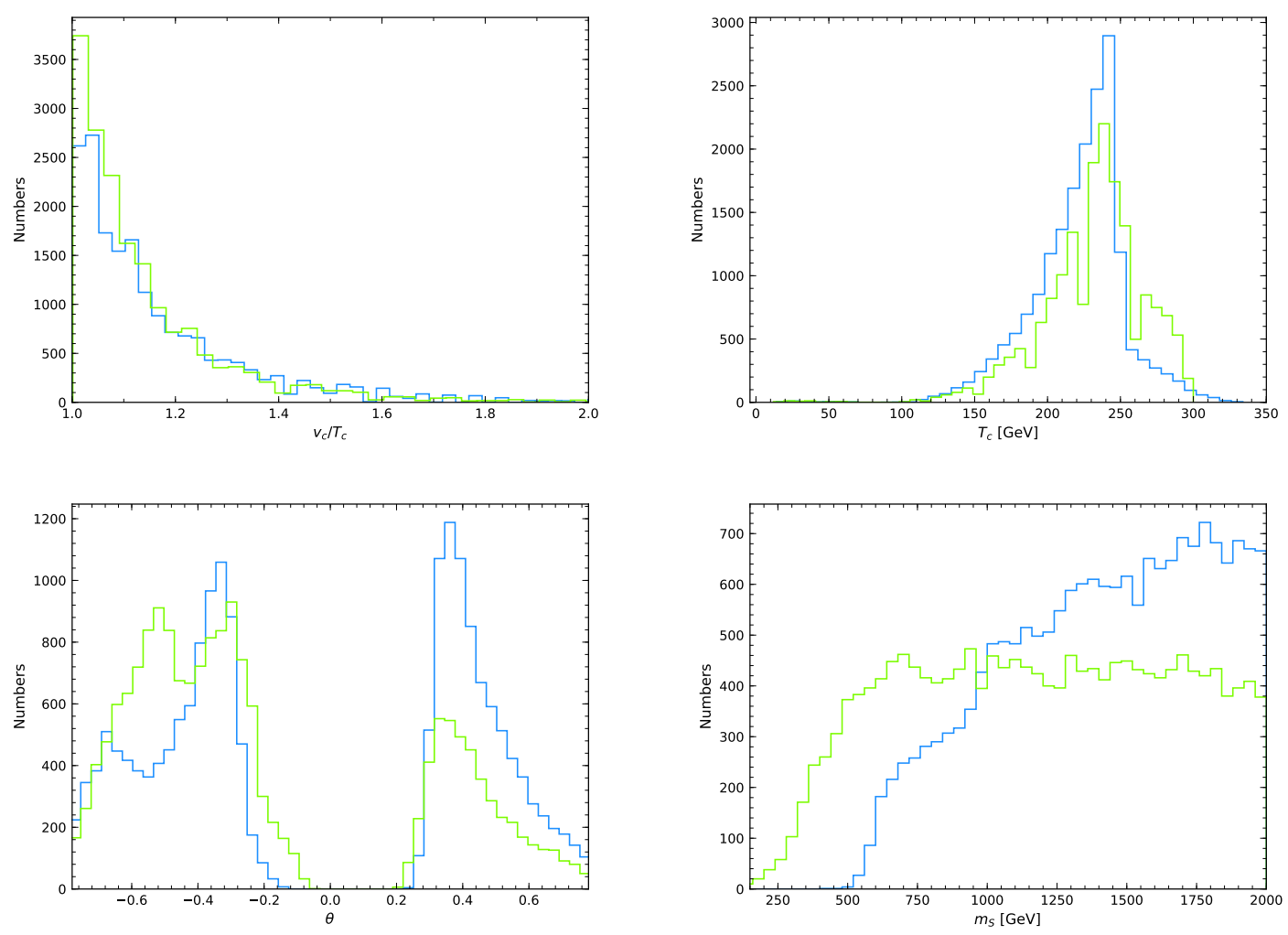

Figure 13. The upper two plots give the distributions in $v_{c} / T_{c}$ and $T_{c}$ and the lower two plots give the distributions in $\theta$ and $m_{\mathcal{S}}$ that can generate a sufficiently strong first-order EWPT. The blue and green histograms represent the random parameter scan results obtained in the Landau gauge potential and the gauge-independent potential, respectively.

$v_{c} / T_{c}$ can be made by retaining only the quadratic temperature-dependent terms in the effective potential (see refs. [123-127] for a systematic treatment of the high-temperature effective theory for a number of SM extensions).

Here we adopt another gauge-invariant approach introduced in ref. [82], which is appropriate for the scenarios in which gauge degrees of freedom play a subdominant role in the generation of potential barrier [82], as in our singlet extension of SM. Following this approach, we truncate the one-loop effective potential $V_{\text {eff }}(T)$ at the second order in the EW gauge couplings but include terms to all orders in the new couplings. Through this procedure, we obtain the gauge-independent effective potential by eliminating the gaugedependent terms that first arise at $\mathcal{O}\left(g^{3}\right)$ [82].

To compare with those results obtained in section 4 by using a Landau gauge, we now search for the first-order EWPT-viable parameter space with the gauge-independent effective potential using the same scheme of random parameter scan described in section 4.2. We show the main results in figure 13. In the figure, the blue histograms are the distributions obtained with a Landau gauge, while the green histograms represent those obtained by using a gauge-independent effective potential, both of them containing about 18000 

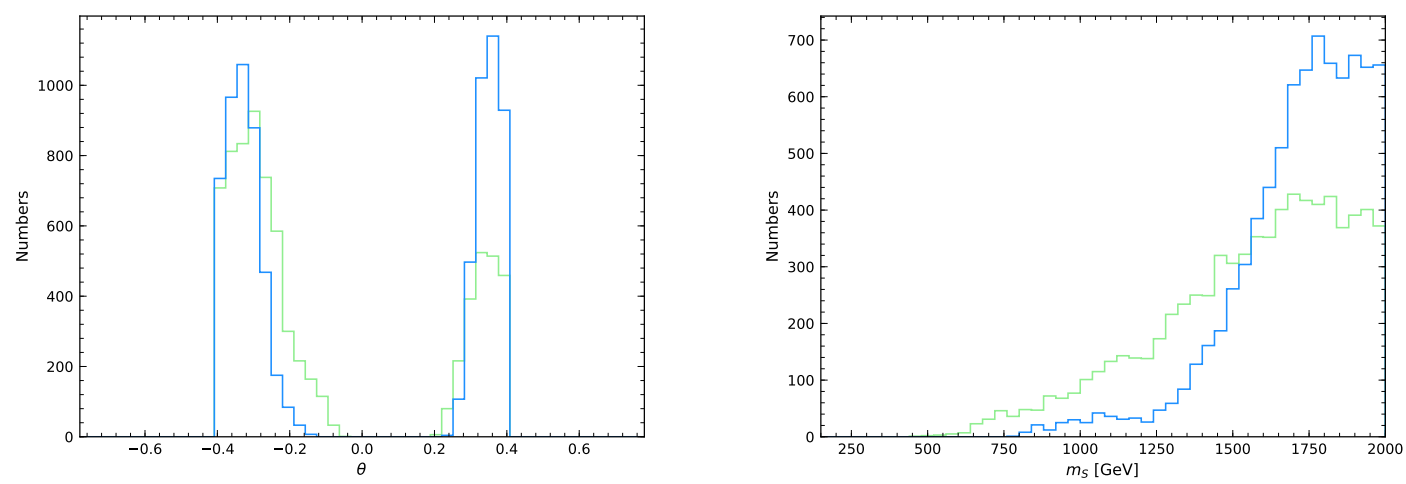

Figure 14. Same as the lower two plots in figure 13 after taking into account the experimental constraints.

samples. The upper two plots of figure 13 show similar distributions in $v_{c} / T_{c}$ and $T_{c}$. From the distribution of $\theta$, we see that the conclusion $|\theta| \gtrsim 0.2$ for a strong first-order EWPT still holds for the gauge-independent potential. However, the lower cutoff in the distribution of $m_{\mathcal{S}}$ is reduced to $\sim 250 \mathrm{GeV}$. The reason for the existence of such a lower bound has been given in section 4.3 .

We now further impose the experimental constraints on the parameter space with the gauge-independent potential, the $\theta$ and $m_{\mathcal{S}}$ distributions are showed in figure 14 (green histograms). As shown in the figure, $0.2 \lesssim|\theta| \lesssim 0.4$ and $1.0 \lesssim m_{\mathcal{S}} \lesssim 2.0 \mathrm{TeV}$ are required for a sufficiently strong EWPT while taking into account the constraints from collider experiments when the gauge-independent potential is used. Hence, our main conclusions from the effective potential using the Landau gauge remain nearly unchanged.

To estimate the impacts of gauge dependence on criterion (4.1), we re-calculate $v_{c}$ and $T_{c}$ in the gauge-independent potential with those samples that satisfy $v_{c} / T_{c} \gtrsim 1$ in the Landau gauge potential, the results are represented by the green histograms in figure 15 . Compare with the random scan results in figure 13, all of the samples are the same here. From the figure we see that for the gauge-independent potential, the distributions in $T_{c}$ and $v_{c} / T_{c}$ mainly fall in the ranges of $\sim 150-250 \mathrm{GeV}$ and $\sim 1.1-1.4$, respectively. For each sample point, we calculate the difference in $T_{c}$ and $v_{c} / T_{c}$ between the gauge-independent potential and the Landau gauge potential, denoted by $\Delta_{T}$ and $\Delta_{R}$, respectively. We show the distributions of $\Delta_{T}$ and $\Delta_{R}$ in the lower plots of figure 15. We find that when the full one-loop potential with the Landau gauge is replaced by the gauge-independent potential, the critical temperature of the phase transition could decrease by $\sim 30 \mathrm{GeV}$ and $v_{c} / T_{c}$ could increase $\sim 0.18$. Thus, if a sample point triggers a first-order EWPT in the full one-loop potential in the Landau gauge, it also does in the gauge-independent potential.

In figure 16, we show the results of GW parameters $\alpha$ and $\beta / H_{*}$ calculated using the gauge-independent potential. All the samples generate a strong first-order EWPT and survive the experimental constraints. As illustrated in this figure, there is a considerable portion of samples that can be detected by BBO and DECIGO. However, in contrast with the results obtained from the Landau gauge potential, relatively few sample points 

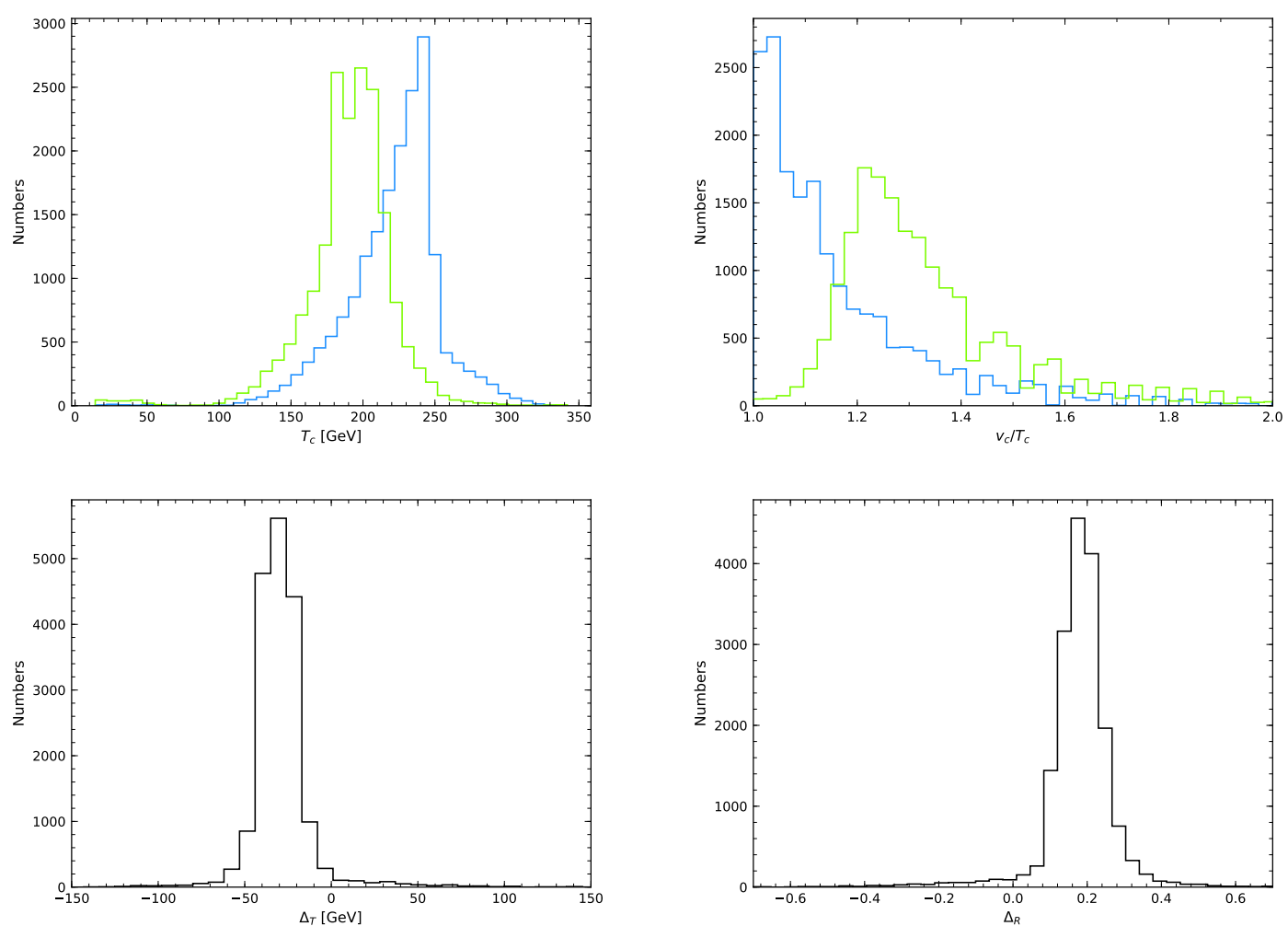

Figure 15. Upper plots: distributions in $T_{c}$ and $v_{c} / T_{c}$ in the Landau gauge potential (blue histogram) and gauge-independent potential (green histogram). Lower plots: distribution of difference between the gauge-independent potential and the Landau gauge potential in $T_{c}$ and $v_{c} / T_{c}$. The samples of input parameters are the same for both of the potential.

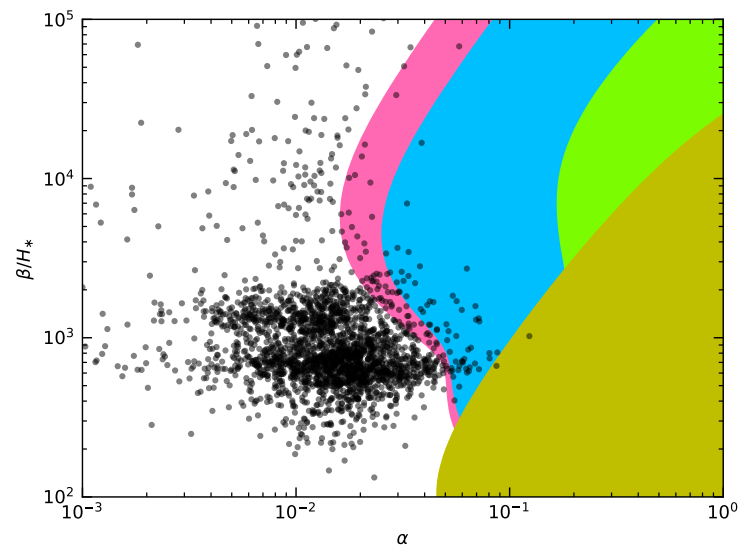

Figure 16. The pink, blue, yellow, and green regions represent respectively the sensitivities of BBO, DECIGO, LISA, and B-DECIGO exceeding the detection threshold, assuming the nucleation temperature $T_{n}=200 \mathrm{GeV}$. The scatter points are the samples that both generate a strong first- order EWPT and survive the collider searches and DM experiments. The gauge-independent potential is adopted for the calculations. 
fall within the sensitivity of LISA. This means that the GWs produced from the firstorder EWPT in the gauge-independent scalar potential may be too weak to be detected by the LISA interferometer. A similar conclusion was also found in ref. [127], in which a nonperturbative analysis of the GW power spectrum was adopted to show that GWs produced from a first-order EWPT in a beyond-the-SM scenario described by a SM-like effective theory will be so weak that the LISA experiment is unable to probe.

\section{Summary and conclusion}

In this work, we have considered the extension of SM with a complex singlet scalar field $S$. A global U(1) symmetry associated with $S$ in the scalar potential is softly broken by its cubic terms to a $\mathbb{Z}_{3}$ symmetry. The real part of the complex scalar field develops a VEV and mixes with the SM Higgs boson after the electroweak symmetry breaking, while the imaginary component, the pseudo-Goldstone boson $\chi$, becomes a DM candidate due to an accidental $\mathbb{Z}_{2}$ symmetry hidden in the scalar potential. We focus on the two-step phase transition scenario, in which the location of global minimum of the potential moves as $(0,0) \rightarrow\left(0, w_{0}(T)\right) \rightarrow(v(T), w(T))$ with the expansion of the Universe. We then search for the parameter space for sample points where a sufficiently strong EWPT can occur, taking into account the constraints from collider experiments and DM searches. The conclusions we have obtained are summarized as follows:

- The requirement of a sufficiently strong EWPT demands $|\theta| \gtrsim 0.2\left(11.5^{\circ}\right)$ for $m_{\mathcal{S}} \lesssim$ $2 \mathrm{TeV}$. The Higgs signal strength measurements, on the other hand, give the constraint $|\theta| \lesssim 0.4\left(23^{\circ}\right)$.

- To trigger a successful phase transition, the real scalar mass $m_{\mathcal{S}}$ should be larger than about $500 \mathrm{GeV}$ (250 GeV in the gauge-independent potential). The constraints of Higgs signal strength measurements further pushes up the lower bound of the scalar mass: $m_{\mathcal{S}} \gtrsim 1.2 \mathrm{TeV}(1.0 \mathrm{TeV}$ in the gauge-independent potential).

- A small fraction of DM could be made of the pseudo-Goldstone boson $\chi$, while the constraints from current DM direct searches are satisfied.

- GW signals from the first-order phase transition in the model with the mixing angle $\theta$ in the range of $\sim 0.25-0.4$ can be detectable using future space-based interferometers, such as DECIGO and BBO.

We concentrate in this work on the two-step phase transition scenario as described in section 4.1. Other types of multi-step phase transitions can be found in recent works $[33,36,85]$. The requirement of a large mixing angle to trigger a sufficiently strong EWPT is crucial in the two-step phase transition. The one-step phase transition, $(0,0) \rightarrow(v(T), w(T))$, can be stronger, and for models with a mixing angle $\lesssim 0.2$, the phase transition may be detectable in planned space-based interferometers [36]. We also note that in our analysis, we have restricted the heavy scalar mass $m_{\mathcal{S}}$ to be in the range of $150-2000 \mathrm{GeV}\left(m_{\mathcal{S}}>m_{\mathcal{H}}\right)$, the strong EWPT with a lower scalar mass, $m_{\mathcal{S}}<m_{\mathcal{H}}$, can be 
found in refs. [84, 85]. Future precision Higgs measurements at collider experiments, such as the high-luminosity LHC, the International Linear Collider, and the Circular ElectronPositron Collider, could further probe the mixing angle regions that trigger a strong firstorder EWPT in our scenario [94]. A more systematic and general analysis of the EWPT and the related collider phenomenology has been given in a recent work [86]. Our scenario for EWPT and its phenomena at the colliders could be a particular illustration of those more general considerations.

\section{Acknowledgments}

We would like to thank E. Senaha and the anonymous Referee for helpful comments and discussions. This work was supported in part by the Ministry of Science and Technology (MOST) of Taiwan under Grant Nos. MOST-104-2628-M-002-014-MY4, MOST-108-2112M-002-005-MY3, MOST-108-2811-M-002-524 and MOST-108-2811-M-002-548.

\section{A Field-dependent mass matrix at finite temperature}

The thermally corrected mass matrix of $h$ and $s$ is

$$
\begin{aligned}
\mathcal{M}^{2}= & \left(\begin{array}{cc}
-\mu_{h}^{2}+3 \lambda_{h} h^{2}+\frac{1}{2} \lambda_{m} s^{2} & \lambda_{m} h s \\
\lambda_{m} h s & \mu_{s}^{2}+3 \lambda_{s} s^{2}+2 \mu_{3} s+\frac{1}{2} \lambda_{m} h^{2}
\end{array}\right) \\
& +\frac{T^{2}}{48}\left(\begin{array}{cc}
9 g^{2}+3 g^{2}+2\left[6\left(y_{t}^{2}+y_{b}^{2}\right)+12 \lambda_{h}+\lambda_{m}\right] & 0 \\
0 & 4\left(2 \lambda_{m}+3 \lambda_{s}\right)
\end{array}\right),
\end{aligned}
$$

where $g$ and $g^{\prime}$ are the SM gauge couplings of $\mathrm{SU}(2)_{L}$ and $\mathrm{U}(1)_{Y}, y_{t}$ and $y_{b}$ are the top and bottom quark Yukawa couplings. The thermally corrected masses of SM Goldstone bosons $G^{0, \pm}$ and pseudo-Goldstone boson $\chi$ are

$$
\begin{aligned}
& \mathcal{M}_{G}^{2}=-\mu_{h}^{2}+\lambda_{h} h^{2}+\frac{1}{2} \lambda_{m} s^{2}+\frac{T^{2}}{48}\left\{9 g^{2}+3 g^{2}+2\left[6\left(y_{t}^{2}+y_{b}^{2}\right)+12 \lambda_{h}+\lambda_{m}\right]\right\}, \\
& \mathcal{M}_{\chi}^{2}=\mu_{s}^{2}+\lambda_{s} s^{2}+\frac{1}{2} \lambda_{m} h^{2}-2 \mu_{3} s+\frac{T^{2}}{12}\left(2 \lambda_{m}+3 \lambda_{s}\right) .
\end{aligned}
$$

The field-dependent masses for the transverse components of SM massive gauge bosons $W$ and $Z$ are

$$
\mathcal{M}_{W, T}^{2}=\frac{1}{4} g^{2} h^{2}, \quad \mathcal{M}_{Z, T}^{2}=\frac{1}{4}\left(g^{2}+g^{\prime 2}\right) h^{2} .
$$

The longitudinal components of $W$ and $Z$ receive thermal corrections from the daisy diagrams, and their masses in terms of $\left(W_{\mu}^{+}, W_{\mu}^{-}, W_{\mu}^{3}, B_{\mu}^{0}\right)$ basis can be written as

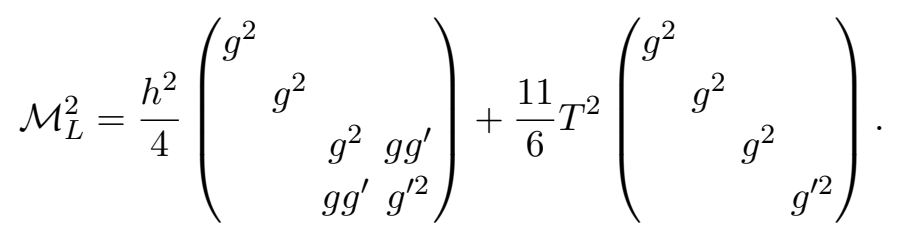

The field-dependent masses of top and bottom quarks are

$$
\mathcal{M}_{t}^{2}=\frac{1}{2} y_{t} h^{2}, \quad \mathcal{M}_{b}^{2}=\frac{1}{2} y_{b} h^{2} .
$$




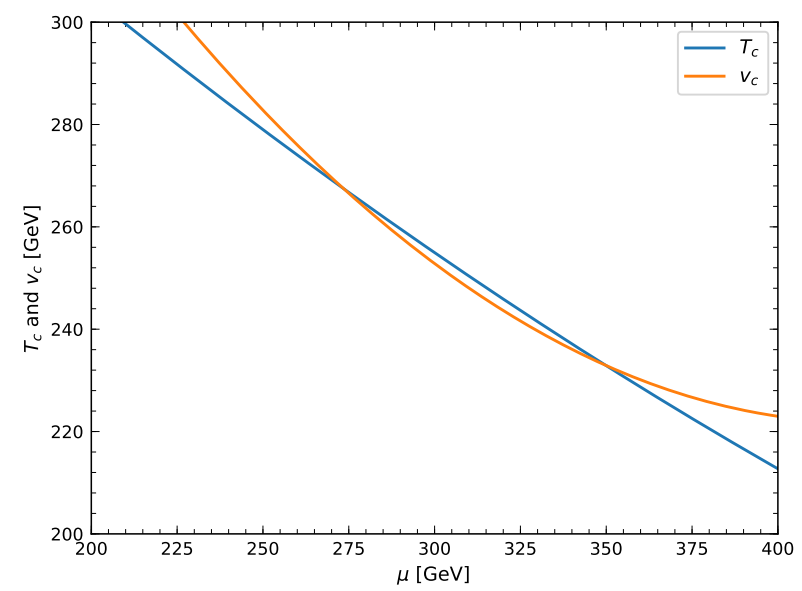

Figure 17. $T_{c}$ and $v_{c}$ as functions of $\mu$, with $w(v)=972.7 \mathrm{GeV}, m_{\mathcal{S}}(v)=655.9 \mathrm{GeV}, m_{\chi}(v)=$ $460.0 \mathrm{GeV}$, and $\theta(v)=0.646$.

\section{B Renormalization group equations}

The RGEs at the one-loop level are [128]

$$
\begin{aligned}
16 \pi^{2} \beta_{\lambda_{h}} & =\lambda_{h}\left(24 \lambda_{h}-\frac{9}{5} g_{1}^{2}-9 g_{2}^{2}+\frac{1}{4} \lambda_{m}^{2}+12 y_{t}^{2}\right)+\frac{27}{200} g_{1}^{4}+\frac{9}{20} g_{1}^{2} g_{2}^{2}+\frac{9}{8} g_{2}^{4}-6 y_{t}^{4}, \\
16 \pi^{2} \beta_{\lambda_{m}} & =\lambda_{m}\left(12 \lambda_{h}-\frac{9}{10} g_{1}^{2}-\frac{9}{2} g_{2}^{2}+2 \lambda_{m}+2 \lambda_{s}+6 y_{t}^{2}\right) \\
16 \pi^{2} \beta_{\lambda_{s}} & =2 \lambda_{m}^{2}+5 \lambda_{s}^{2} \\
16 \pi^{2} \beta_{\mu_{h}^{2}} & =\mu_{h}^{2}\left(12 \lambda_{h}-\frac{9}{10} g_{1}^{2}-\frac{9}{2} g_{2}^{2}+6 y_{t}^{2}\right)-\frac{1}{2} \lambda_{m} \mu_{s}^{2} \\
16 \pi^{2} \beta_{\mu_{s}^{2}} & =2 \mu_{3}^{2}-4 \lambda_{m} \mu_{h}^{2}+2 \lambda_{s} \mu_{s}^{2} \\
16 \pi^{2} \beta_{\mu_{3}} & =3 \lambda_{s} \mu_{3} .
\end{aligned}
$$

The parameters in the tree-level potential and $V_{\mathrm{CW}}$ have a dependence on the renormalization scale $\mu$, and can lead to a significant $\mu$-dependence in $T_{c}$ and $v_{c}$. We have fixed the value of renormalization scale $\mu=v$ in the results presented in the main text.

In figure 17 we estimate the $\mu$-dependence of $T_{c}$ and $v_{c}$ by way of example. To do this, we first take a sample point of the input parameters: $w(v)=972.7 \mathrm{GeV}, m_{\mathcal{S}}(v)=$ $655.9 \mathrm{GeV}, m_{\chi}(v)=460.0 \mathrm{GeV}$, and $\theta(v)=0.646$ and determine the parameters at another renormalization scale $\mu$ using eqs. (B.1)-(B.6). We then use these parameters to calculate an RGI potentials. Following the procedure described in section 4.2 , we determine $T_{c}(\mu)$ and $v_{c}(\mu)$, as shown in figure 17 . We observe that both $T_{c}$ and $v_{c}$ decrease with $\mu$, while the ratio $v_{c} / T_{c}$ is seen to have much less dependence on the renormalization scale. 


\section{Counter-terms}

To maintain the main properties of the tree-level potential derived in the content, we add a counter-terms (3.5) to renormalize the potential at zero temperature. The renormalization conditions we used are given by

$$
\begin{aligned}
\left.\left(\frac{\partial}{\partial h}, \frac{\partial}{\partial s}, \frac{\partial^{2}}{\partial h^{2}}, \frac{\partial^{2}}{\partial s^{2}}, \frac{\partial^{2}}{\partial h \partial s}\right)\left(V_{\mathrm{CW}}+V_{\mathrm{CT}}\right)\right|_{(h, s)=(v, w)} & =0 \\
\left.\frac{\partial}{\partial s}\left(V_{\mathrm{CW}}+V_{\mathrm{CT}}\right)\right|_{(h, s)=\left(v, w^{\prime}\right)} & =0
\end{aligned}
$$

where $w^{\prime}=s_{-}$is determined by eq. (2.15). With

$$
\begin{array}{lll}
F_{10}=\left.\frac{\partial}{\partial h} V_{\mathrm{CW}}(h, s)\right|_{(h, s)=(v, w)}, & F_{01}=\left.\frac{\partial}{\partial s} V_{\mathrm{CW}}(h, s)\right|_{(h, s)=(v, w)}, \\
F_{01}^{\prime}=\left.\frac{\partial}{\partial s} V_{\mathrm{CW}}(h, s)\right|_{(h, s)=\left(v, w^{\prime}\right)}, & F_{11}=\left.\frac{\partial^{2}}{\partial h \partial s} V_{\mathrm{CW}}(h, s)\right|_{(h, s)=(v, w)}, \\
F_{20}=\left.\frac{\partial^{2}}{\partial h^{2}} V_{\mathrm{CW}}(h, s)\right|_{(h, s)=(v, w)}, & F_{02}=\left.\frac{\partial^{2}}{\partial s^{2}} V_{\mathrm{CW}}(h, s)\right|_{(h, s)=(v, w)},
\end{array}
$$

the renormalization conditions fix the counter-terms as

$$
\begin{aligned}
\delta u_{h}^{2}= & \frac{1}{2 v}\left(-3 F_{10}+w F_{11}+v F_{20}\right) \\
\delta \lambda_{h}= & -\frac{1}{2 v^{3}}\left(-F_{10}+v F_{20}\right) \\
\delta \lambda_{m}= & -\frac{1}{v w} F_{11} \\
\delta \mu_{s}^{2}= & \frac{1}{2 w w^{\prime}\left(w-w^{\prime}\right)^{2}}\left(4 w^{\prime 3} F_{01}-6 w w^{\prime 2} F_{01}-v w^{\prime 3} F_{11}\right. \\
& \left.+2 v w w^{\prime 2} F_{11}-2 w w^{\prime 3} F_{02}-v w^{2} w^{\prime} F_{11}+2 w^{2} w^{\prime 2} F_{02}+2 w^{3} F_{01}^{\prime}\right), \\
\delta \mu_{3}= & \frac{1}{w^{2} w^{\prime}\left(w-w^{\prime}\right)^{2}}\left(-w^{3} F_{01}+3 w^{\prime} w^{2} F_{01}-2 w^{3} F_{01}^{\prime}+w w^{\prime 3} F_{02}-w^{\prime} w^{3} F_{02}\right), \\
\delta \lambda_{s}= & \frac{1}{w^{2} w^{\prime}\left(w-w^{\prime}\right)^{2}}\left(w^{\prime 2} F_{01}-2 w w^{\prime} F_{01}-w w^{\prime 2} F_{02}+w^{\prime} w^{2} F_{02}+w^{2} F_{01}^{\prime}\right) .
\end{aligned}
$$

\section{Decay width}

Here we give the partial width formulas for some decays of SM-like Higgs $\mathcal{H}$ and heavy scalar $\mathcal{S}$ :

$$
\begin{aligned}
& \Gamma_{\mathcal{H} \rightarrow \chi \chi}=\frac{g_{\mathcal{H} \chi \chi}^{2}}{8 \pi m_{\mathcal{H}}} \sqrt{1-\frac{4 m_{\chi}^{2}}{m_{\mathcal{H}}^{2}}}, \quad \Gamma_{\mathcal{H} \rightarrow \mathcal{S S}}=\frac{g_{\mathcal{H} \mathcal{S}}^{2}}{8 \pi m_{\mathcal{H}}} \sqrt{1-\frac{4 m_{\mathcal{S}}^{2}}{m_{\mathcal{H}}^{2}}} \\
& \Gamma_{\mathcal{S} \rightarrow \chi \chi}=\frac{g_{\mathcal{S} \chi \chi}^{2}}{8 \pi m_{\mathcal{S}}} \sqrt{1-\frac{4 m_{\chi}^{2}}{m_{\mathcal{S}}^{2}}}, \quad \Gamma_{\mathcal{S} \rightarrow \mathcal{H H}}=\frac{g_{\mathcal{S H H}}^{2}}{8 \pi m_{\mathcal{S}}} \sqrt{1-\frac{4 m_{\mathcal{H}}^{2}}{m_{\mathcal{S}}^{2}}}
\end{aligned}
$$


where the couplings

$$
\begin{aligned}
g_{\mathcal{H} \chi \chi}= & -2\left(\mu_{3}-\lambda_{s} w\right) \sin \theta-\lambda_{m} v \cos \theta, \\
g_{\mathcal{S} \chi \chi}= & 2\left(\mu_{3}-\lambda_{s} w\right) \cos \theta-\lambda_{m} v \sin \theta, \\
g_{\mathcal{H S S}}= & -\lambda_{m} v \cos ^{3} \theta+2 \cos ^{2} \theta \sin \theta\left(\mu_{3}-\lambda_{m} w+3 \lambda_{s} w\right) \\
& +\sin ^{2} \theta\left[2 \cos \theta\left(-3 \lambda_{h}+\lambda_{m}\right)+\lambda_{m} w \sin \theta\right], \\
g_{\mathcal{S H \mathcal { H }}}= & -\lambda_{m} v \cos ^{3} \theta-2 v \cos ^{2} \theta \sin \theta\left(3 \lambda_{h}-\lambda_{m}\right) \\
& -\sin ^{2} \theta\left[2 \cos \theta\left(\mu_{3}-\lambda_{m} w+3 \lambda_{s} w\right)+\lambda_{m} v \sin \theta\right] .
\end{aligned}
$$

\section{E Sensitivity of space-based interferometers}

Here we summarize the experimental noise power spectral density used in this work. More details can be found in ref. [37] and references therein.

The LISA interferometer is planned to launch in 2034, with the noise power spectral density well approximated by $[62,119]$

$$
S_{\mathrm{LISA}}(f)=\frac{10}{3 L^{2}}\left(P_{\mathrm{OMS}}(f)+2\left[1+\cos ^{2}\left(\frac{f}{f_{*}}\right)\right] \frac{P_{\mathrm{acc}}(f)}{(2 \pi f)^{4}}\right)\left[1+\frac{6}{10}\left(\frac{f}{f_{*}}\right)^{2}\right]+S_{c}(f),
$$

where $L=2.5 \times 10^{9} \mathrm{~m}$ is the arm length of the LISA detectors and the transfer frequency $f_{*}=c /(2 \pi L)$, where $c$ is the speed of light. The instrument noise consists of the optical metrology noise

$$
P_{\mathrm{OMS}}(f)=\left(1.5 \times 10^{-11} \mathrm{~m}\right)^{2}\left[1+\left(\frac{2 \mathrm{mHz}}{f}\right)^{4}\right] \mathrm{Hz}^{-1}
$$

and the test mass acceleration noise

$$
P_{\text {acc }}(f)=\left(3 \times 10^{-15} \mathrm{~m} \mathrm{sec}^{-2}\right)^{2}\left[1+\left(\frac{0.4 \mathrm{mHz}}{f}\right)^{2}\right]\left[1+\left(\frac{f}{8 \mathrm{mHz}}\right)^{4}\right] \mathrm{Hz}^{-1} .
$$

The last term is the confusion noise from unresolved galactic binaries (4 years)

$$
\begin{aligned}
S_{c}(f)= & 9 \times 10^{-45} f^{-7 / 3}\left\{1+\tanh \left[2.184-\left(\frac{1680 f}{\mathrm{~Hz}}\right)\right]\right\} \\
& \times \exp \left[-\left(\frac{f}{\mathrm{~Hz}}\right)^{0.138}-\left(\frac{221 f}{\mathrm{~Hz}}\right) \sin \left(\frac{521 f}{\mathrm{~Hz}}\right)\right] \mathrm{Hz}^{-1} .
\end{aligned}
$$

The B-DECIGO as a scaled-down predecessor of DECIGO is planned to launch in 2020 [63]. Its noise power spectral density can be approximated by [120]

$$
S_{\mathrm{B}-\mathrm{DECIGO}}(f)=2.02 \times 10^{-48}\left[10^{3}+15.84\left(\frac{f}{\mathrm{~Hz}}\right)^{-4}+1.584\left(\frac{f}{\mathrm{~Hz}}\right)^{2}\right] \mathrm{Hz}^{-1} .
$$


The DECIGO and BBO noise power spectral densities can be parameterized as $[121,122]$

$$
S_{\text {DECIGO }, \mathrm{BBO}}(f)=\min \left[\frac{S_{n}^{\text {inst }}(f)}{\exp \left(-\kappa \mathcal{T}_{\mathrm{obs}}^{-1} d N / d f\right)}, S_{n}^{\text {inst }}(f)+S_{n}^{\text {gal }}(f) \mathcal{F}(f)\right]+S_{n}^{\text {ex }- \text { gal }} \mathcal{F}(f),(\text { E. } 6)
$$

where $\mathcal{T}_{\text {obs }}$ is the duration of the mission, $\kappa=5, d N / d f=2 \times(f / \mathrm{Hz})^{-11 / 3} \mathrm{~Hz}^{-1}$ is the spectral number density of galactic white dwarf binaries, and the factor $\mathcal{F}(f) \equiv$ $\exp \left[-2(f / 0.05 \mathrm{~Hz})^{2}\right]$ corresponds to the high frequency cutoff for the white dwarf confusion noises. The non sky-averaged instrumental noise spectral density for DECIGO and $\mathrm{BBO}$ are respectively

$$
\begin{aligned}
S_{n, \mathrm{DECIGO}}^{\text {inst }}(f)= & 5.3 \times 10^{-48}\left[1+\left(\frac{f}{f_{p}}\right)^{2}+\frac{2.3 \times 10^{-7}}{1+\left(f / f_{p}\right)^{2}}\left(\frac{f}{f_{p}}\right)^{-4}\right. \\
& \left.+2.6 \times 10^{-8}\left(\frac{f}{f_{p}}\right)^{-4}\right] \mathrm{Hz}^{-1}, \\
S_{n, \mathrm{BBO}}^{\text {inst }}(f)= & 10^{-49}\left[1.8\left(\frac{f}{\mathrm{~Hz}}\right)^{2}+2.9+9.2 \times 10^{-3}\left(\frac{f}{\mathrm{~Hz}}\right)^{-4}\right] \mathrm{Hz}^{-1},
\end{aligned}
$$

where $f_{p}=7.36 \mathrm{~Hz}$. The confusion noises from galactic and extra-galactic white dwarf binaries are given respectively by

$$
\begin{aligned}
S_{n}^{\text {gal }} & =2.1 \times 10^{-45}\left(\frac{f}{\mathrm{~Hz}}\right)^{-7 / 3} \mathrm{~Hz}^{-1}, \\
S_{n}^{\text {ex }- \text { gal }} & =4.2 \times 10^{-47}\left(\frac{f}{\mathrm{~Hz}}\right)^{-7 / 3} \mathrm{~Hz}^{-1} .
\end{aligned}
$$

The experimental frequency range, duration of the mission and other parameters for the future GW interferometers are summarized in table 2 .

Open Access. This article is distributed under the terms of the Creative Commons Attribution License (CC-BY 4.0), which permits any use, distribution and reproduction in any medium, provided the original author(s) and source are credited.

\section{References}

[1] ATLAS collaboration, Observation of a new particle in the search for the Standard Model Higgs boson with the ATLAS detector at the LHC, Phys. Lett. B 716 (2012) 1 [arXiv:1207.7214] [INSPIRE].

[2] CMS collaboration, Observation of a New Boson at a Mass of $125 \mathrm{GeV}$ with the CMS Experiment at the LHC, Phys. Lett. B $\mathbf{7 1 6}$ (2012) 30 [arXiv:1207.7235] [INSPIRE].

[3] Planck collaboration, Planck 2015 results. XIII. Cosmological parameters, Astron. Astrophys. 594 (2016) A13 [arXiv:1502.01589] [inSPIRE].

[4] V.A. Kuzmin, V.A. Rubakov and M.E. Shaposhnikov, On the Anomalous Electroweak Baryon Number Nonconservation in the Early Universe, Phys. Lett. B 155 (1985) 36 [INSPIRE]. 
[5] A.G. Cohen, D.B. Kaplan and A.E. Nelson, Weak scale baryogenesis, Phys. Lett. B 245 (1990) 561 [INSPIRE].

[6] J.M. Cline, Status of electroweak phase transition and baryogenesis, Pramana 55 (2000) 33 [hep-ph/0003029] [INSPIRE].

[7] D.E. Morrissey and M.J. Ramsey-Musolf, Electroweak baryogenesis, New J. Phys. 14 (2012) 125003 [arXiv: 1206.2942] [INSPIRE].

[8] J.M. Cline, Baryogenesis, in Les Houches Summer School - Session 86: Particle Physics and Cosmology: The Fabric of Spacetime, Les Houches France (2006) [hep-ph/0609145] [INSPIRE].

[9] M. D'Onofrio, K. Rummukainen and A. Tranberg, Sphaleron Rate in the Minimal Standard Model, Phys. Rev. Lett. 113 (2014) 141602 [arXiv:1404.3565] [INSPIRE].

[10] F. Zwicky, On the Masses of Nebulae and of Clusters of Nebulae, Astrophys. J. 86 (1937) 217 [INSPIRE].

[11] V.C. Rubin and J. Ford, W.Kent, Rotation of the Andromeda Nebula from a Spectroscopic Survey of Emission Regions, Astrophys. J. 159 (1970) 379 [INSPIRE].

[12] R. Massey et al., Dark matter maps reveal cosmic scaffolding, Nature 445 (2007) 286 [astro-ph/0701594] [INSPIRE].

[13] B.W. Lee and S. Weinberg, Cosmological Lower Bound on Heavy Neutrino Masses, Phys. Rev. Lett. 39 (1977) 165 [inSPIRE].

[14] P. Hut, Limits on Masses and Number of Neutral Weakly Interacting Particles, Phys. Lett. $B 69$ (1977) 85 [INSPIRE].

[15] E. Ma, Z(3) Dark Matter and Two-Loop Neutrino Mass, Phys. Lett. B 662 (2008) 49 [arXiv:0708.3371] [INSPIRE].

[16] V. Barger, P. Langacker, M. McCaskey, M. Ramsey-Musolf and G. Shaughnessy, Complex Singlet Extension of the Standard Model, Phys. Rev. D 79 (2009) 015018 [arXiv:0811.0393] [INSPIRE].

[17] M. Gonderinger, H. Lim and M.J. Ramsey-Musolf, Complex Scalar Singlet Dark Matter: Vacuum Stability and Phenomenology, Phys. Rev. D 86 (2012) 043511 [arXiv:1202.1316] [INSPIRE].

[18] M. Jiang, L. Bian, W. Huang and J. Shu, Impact of a complex singlet: Electroweak baryogenesis and dark matter, Phys. Rev. D 93 (2016) 065032 [arXiv:1502.07574] [INSPIRE].

[19] C.-W. Chiang, M.J. Ramsey-Musolf and E. Senaha, Standard Model with a Complex Scalar Singlet: Cosmological Implications and Theoretical Considerations, Phys. Rev. D 97 (2018) 015005 [arXiv: 1707.09960] [INSPIRE].

[20] C.E. Yaguna, Gamma rays from the annihilation of singlet scalar dark matter, JCAP 03 (2009) 003 [arXiv:0810.4267] [INSPIRE].

[21] S. Profumo, L. Ubaldi and C. Wainwright, Singlet Scalar Dark Matter: monochromatic gamma rays and metastable vacua, Phys. Rev. D 82 (2010) 123514 [arXiv:1009.5377] [INSPIRE].

[22] C. Arina and M.H.G. Tytgat, Constraints on Light WIMP candidates from the Isotropic Diffuse Gamma-Ray Emission, JCAP 01 (2011) 011 [arXiv:1007.2765] [INSPIRE]. 
[23] Y. Mambrini, Higgs searches and singlet scalar dark matter: Combined constraints from XENON 100 and the LHC, Phys. Rev. D 84 (2011) 115017 [arXiv:1108.0671] [InSPIRE].

[24] J.M. Cline, K. Kainulainen, P. Scott and C. Weniger, Update on scalar singlet dark matter, Phys. Rev. D 88 (2013) 055025 [Erratum ibid. 92 (2015) 039906] [arXiv:1306.4710] [INSPIRE].

[25] S. Bhattacharya, S. Jana and S. Nandi, Neutrino Masses and Scalar Singlet Dark Matter, Phys. Rev. D 95 (2017) 055003 [arXiv: 1609.03274] [InSPIRE].

[26] J.A. Casas, D.G. Cerdeño, J.M. Moreno and J. Quilis, Reopening the Higgs portal for single scalar dark matter, JHEP 05 (2017) 036 [arXiv: 1701.08134] [INSPIRE].

[27] GAMBIT collaboration, Status of the scalar singlet dark matter model, Eur. Phys. J. C 77 (2017) 568 [arXiv: 1705.07931] [INSPIRE].

[28] S. Baek, P. Ko and W.-I. Park, Search for the Higgs portal to a singlet fermionic dark matter at the LHC, JHEP 02 (2012) 047 [arXiv: 1112.1847] [INSPIRE].

[29] M. Fairbairn and R. Hogan, Singlet Fermionic Dark Matter and the Electroweak Phase Transition, JHEP 09 (2013) 022 [arXiv: 1305.3452] [INSPIRE].

[30] T. Li and Y.-F. Zhou, Strongly first order phase transition in the singlet fermionic dark matter model after LUX, JHEP 07 (2014) 006 [arXiv:1402.3087] [INSPIRE].

[31] A. Beniwal, M. Lewicki, J.D. Wells, M. White and A.G. Williams, Gravitational wave, collider and dark matter signals from a scalar singlet electroweak baryogenesis, JHEP 08 (2017) 108 [arXiv: 1702.06124] [INSPIRE].

[32] A. Beniwal, M. Lewicki, M. White and A.G. Williams, Gravitational waves and electroweak baryogenesis in a global study of the extended scalar singlet model, JHEP 02 (2019) 183 [arXiv: 1810.02380] [INSPIRE].

[33] K. Hashino, M. Kakizaki, S. Kanemura, P. Ko and T. Matsui, Gravitational waves from first order electroweak phase transition in models with the $\mathrm{U}(1)_{X}$ gauge symmetry, JHEP 06 (2018) 088 [arXiv: 1802.02947] [INSPIRE].

[34] C.-W. Chiang and E. Senaha, On gauge dependence of gravitational waves from a first-order phase transition in classical scale-invariant $\mathrm{U}(1)^{\prime}$ models, Phys. Lett. $B 774$ (2017) 489 [arXiv: 1707.06765] [INSPIRE].

[35] A. Ahriche, K. Hashino, S. Kanemura and S. Nasri, Gravitational Waves from Phase Transitions in Models with Charged Singlets, Phys. Lett. B 789 (2019) 119 [arXiv: 1809.09883] [INSPIRE].

[36] A. Alves, T. Ghosh, H.-K. Guo, K. Sinha and D. Vagie, Collider and Gravitational Wave Complementarity in Exploring the Singlet Extension of the Standard Model, JHEP 04 (2019) 052 [arXiv: 1812.09333] [INSPIRE].

[37] M. Breitbach, J. Kopp, E. Madge, T. Opferkuch and P. Schwaller, Dark, Cold and Noisy: Constraining Secluded Hidden Sectors with Gravitational Waves, JCAP 07 (2019) 007 [arXiv:1811.11175] [INSPIRE].

[38] M. Duch, B. Grzadkowski and M. McGarrie, A stable Higgs portal with vector dark matter, JHEP 09 (2015) 162 [arXiv:1506.08805] [INSPIRE].

[39] X.-G. He and J. Tandean, New LUX and PandaX-II Results Illuminating the Simplest Higgs-Portal Dark Matter Models, JHEP 12 (2016) 074 [arXiv:1609.03551] [INSPIRE]. 
[40] K. Ghorbani and P.H. Ghorbani, Strongly First-Order Phase Transition in Real Singlet Scalar Dark Matter Model, J. Phys. G 47 (2020) 015201 [arXiv: 1804.05798] [INSPIRE].

[41] P.S.B. Dev, F. Ferrer, Y. Zhang and Y. Zhang, Gravitational Waves from First-Order Phase Transition in a Simple Axion-Like Particle Model, JCAP 11 (2019) 006 [arXiv: 1905.00891] [INSPIRE].

[42] A. Paul, B. Banerjee and D. Majumdar, Gravitational wave signatures from an extended inert doublet dark matter model, JCAP 10 (2019) 062 [arXiv:1908.00829] [INSPIRE].

[43] T. Alanne, T. Hugle, M. Platscher and K. Schmitz, A fresh look at the gravitational-wave signal from cosmological phase transitions, JHEP 03 (2020) 004 [arXiv:1909.11356] [INSPIRE].

[44] W. Chao, H.-K. Guo and J. Shu, Gravitational Wave Signals of Electroweak Phase Transition Triggered by Dark Matter, JCAP 09 (2017) 009 [arXiv: 1702. 02698] [InSPIRE].

[45] B. Grzadkowski and D. Huang, Spontaneous CP-Violating Electroweak Baryogenesis and Dark Matter from a Complex Singlet Scalar, JHEP 08 (2018) 135 [arXiv:1807.06987] [INSPIRE].

[46] Z. Kang, P. Ko and T. Matsui, Strong first order EWPT \& strong gravitational waves in $Z_{3}$-symmetric singlet scalar extension, JHEP 02 (2018) 115 [arXiv:1706.09721] [INSPIRE].

[47] K. Kannike, K. Loos and M. Raidal, Gravitational wave signals of pseudo-Goldstone dark matter in the $\mathbb{Z}_{3}$ complex singlet model, Phys. Rev. D 101 (2020) 035001 [arXiv:1907.13136] [INSPIRE].

[48] S. Profumo, M.J. Ramsey-Musolf and G. Shaughnessy, Singlet Higgs phenomenology and the electroweak phase transition, JHEP 08 (2007) 010 [arXiv:0705.2425] [INSPIRE].

[49] J.R. Espinosa, T. Konstandin, J.M. No and M. Quirós, Some Cosmological Implications of Hidden Sectors, Phys. Rev. D 78 (2008) 123528 [arXiv:0809.3215] [INSPIRE].

[50] K. Kannike and M. Raidal, Phase Transitions and Gravitational Wave Tests of Pseudo-Goldstone Dark Matter in the Softly Broken U(1) Scalar Singlet Model, Phys. Rev. D 99 (2019) 115010 [arXiv:1901.03333] [INSPIRE].

[51] G. Kurup and M. Perelstein, Dynamics of Electroweak Phase Transition In Singlet-Scalar Extension of the Standard Model, Phys. Rev. D 96 (2017) 015036 [arXiv:1704.03381] [INSPIRE].

[52] LUX collaboration, Results from a search for dark matter in the complete LUX exposure, Phys. Rev. Lett. 118 (2017) 021303 [arXiv: 1608.07648] [INSPIRE].

[53] PandaX-II collaboration, Dark Matter Results From 54-Ton-Day Exposure of PandaX-II Experiment, Phys. Rev. Lett. 119 (2017) 181302 [arXiv:1708.06917] [INSPIRE].

[54] XENON collaboration, Dark Matter Search Results from a One Ton-Year Exposure of XENON1T, Phys. Rev. Lett. 121 (2018) 111302 [arXiv:1805.12562] [INSPIRE].

[55] V. Barger, P. Langacker, M. McCaskey, M.J. Ramsey-Musolf and G. Shaughnessy, LHC Phenomenology of an Extended Standard Model with a Real Scalar Singlet, Phys. Rev. D 77 (2008) 035005 [arXiv:0706.4311] [INSPIRE].

[56] Y. Cai, X.-G. He and B. Ren, Low Mass Dark Matter and Invisible Higgs Width In Darkon Models, Phys. Rev. D 83 (2011) 083524 [arXiv:1102.1522] [INSPIRE]. 
[57] C. Gross, O. Lebedev and T. Toma, Cancellation Mechanism for Dark-Matter-Nucleon Interaction, Phys. Rev. Lett. 119 (2017) 191801 [arXiv:1708.02253] [INSPIRE].

[58] X.-G. He, T. Li, X.-Q. Li, J. Tandean and H.-C. Tsai, Constraints on Scalar Dark Matter from Direct Experimental Searches, Phys. Rev. D 79 (2009) 023521 [arXiv:0811.0658] [INSPIRE].

[59] J.M. Cline and K. Kainulainen, Electroweak baryogenesis and dark matter from a singlet Higgs, JCAP 01 (2013) 012 [arXiv:1210.4196] [INSPIRE].

[60] D. Azevedo, M. Duch, B. Grzadkowski, D. Huang, M. Iglicki and R. Santos, One-loop contribution to dark-matter-nucleon scattering in the pseudo-scalar dark matter model, JHEP 01 (2019) 138 [arXiv: 1810.06105] [INSPIRE].

[61] K. Ishiwata and T. Toma, Probing pseudo Nambu-Goldstone boson dark matter at loop level, JHEP 12 (2018) 089 [arXiv:1810.08139] [INSPIRE].

[62] LISA collaboration, Laser Interferometer Space Antenna, arXiv:1702.00786 [INSPIRE].

[63] S. Sato et al., The status of DECIGO, J. Phys. Conf. Ser. 840 (2017) 012010 [InSPIRE].

[64] J. Crowder and N.J. Cornish, Beyond LISA: Exploring future gravitational wave missions, Phys. Rev. D 72 (2005) 083005 [gr-qc/0506015] [inSPIRE].

[65] Y. Zeldovich, I. Kobzarev and L.B. Okun, Cosmological Consequences of the Spontaneous Breakdown of Discrete Symmetry, Zh. Eksp. Teor. Fiz. 67 (1974) 3 [inSPIRE].

[66] T.W.B. Kibble, Topology of Cosmic Domains and Strings, J. Phys. A 9 (1976) 1387 [INSPIRE].

[67] P. Sikivie, Of Axions, Domain Walls and the Early Universe, Phys. Rev. Lett. 48 (1982) 1156 [INSPIRE].

[68] A. Vilenkin, Cosmic strings and domain walls, Phys. Rept. 121 (1985) 5 [INSPIRE].

[69] G.B. Gelmini, M. Gleiser and E.W. Kolb, Cosmology of Biased Discrete Symmetry Breaking, Phys. Rev. D 39 (1989) 1558 [InSPIRE].

[70] J. Preskill, S.P. Trivedi, F. Wilczek and M.B. Wise, Cosmology and broken discrete symmetry, Nucl. Phys. B 363 (1991) 207 [InSPIRE].

[71] S.A. Abel, S. Sarkar and P.L. White, On the cosmological domain wall problem for the minimally extended supersymmetric standard model, Nucl. Phys. B 454 (1995) 663 [hep-ph/9506359] [INSPIRE].

[72] C. Panagiotakopoulos and K. Tamvakis, Stabilized NMSSM without domain walls, Phys. Lett. B 446 (1999) 224 [hep-ph/9809475] [INSPIRE].

[73] T. Hiramatsu, M. Kawasaki and K. Saikawa, On the estimation of gravitational wave spectrum from cosmic domain walls, JCAP 02 (2014) 031 [arXiv:1309.5001] [INSPIRE].

[74] R. Zhou, J. Yang and L. Bian, Gravitational Waves from first-order phase transition and domain wall, JHEP 04 (2020) 071 [arXiv: 2001.04741] [INSPIRE].

[75] J.R. Espinosa, T. Konstandin and F. Riva, Strong Electroweak Phase Transitions in the Standard Model with a Singlet, Nucl. Phys. B 854 (2012) 592 [arXiv:1107.5441] [inSPIRE].

[76] S. Kanemura, M. Kikuchi and K. Yagyu, Radiative corrections to the Higgs boson couplings in the model with an additional real singlet scalar field, Nucl. Phys. B 907 (2016) 286 [arXiv:1511.06211] [INSPIRE]. 
[77] S.R. Coleman and E.J. Weinberg, Radiative Corrections as the Origin of Spontaneous Symmetry Breaking, Phys. Rev. D 7 (1973) 1888 [INSPIRE].

[78] M.E. Carrington, The Effective potential at finite temperature in the Standard Model, Phys. Rev. D 45 (1992) 2933 [InSPIRE].

[79] R. Jackiw, Functional evaluation of the effective potential, Phys. Rev. D 9 (1974) 1686 [INSPIRE].

[80] H.H. Patel and M.J. Ramsey-Musolf, Baryon Washout, Electroweak Phase Transition and Perturbation Theory, JHEP 07 (2011) 029 [arXiv:1101.4665] [INSPIRE].

[81] C. Wainwright, S. Profumo and M.J. Ramsey-Musolf, Gravity Waves from a Cosmological Phase Transition: Gauge Artifacts and Daisy Resummations, Phys. Rev. D 84 (2011) 023521 [arXiv: 1104.5487] [INSPIRE].

[82] A. Katz, M. Perelstein, M.J. Ramsey-Musolf and P. Winslow, Stop-Catalyzed Baryogenesis Beyond the MSSM, Phys. Rev. D 92 (2015) 095019 [arXiv: 1509. 02934] [InSPIRE].

[83] L. Dolan and R. Jackiw, Symmetry Behavior at Finite Temperature, Phys. Rev. D 9 (1974) 3320 [INSPIRE].

[84] J. Kozaczuk, M.J. Ramsey-Musolf and J. Shelton, Exotic Higgs Decays and the Electroweak Phase Transition, Phys. Rev. D 101 (2020) 115035 [arXiv:1911.10210] [InSPIRE].

[85] M. Carena, Z. Liu and Y. Wang, Electroweak Phase Transition with Spontaneous $Z_{2}$-Breaking, arXiv:1911.10206 [INSPIRE].

[86] M.J. Ramsey-Musolf, The Electroweak Phase Transition: A Collider Target, arXiv: 1912.07189 [INSPIRE].

[87] G.M. Pruna and T. Robens, Higgs singlet extension parameter space in the light of the LHC discovery, Phys. Rev. D 88 (2013) 115012 [arXiv:1303.1150] [INSPIRE].

[88] J.M. Cline, G. Laporte, H. Yamashita and S. Kraml, Electroweak Phase Transition and LHC Signatures in the Singlet Majoron Model, JHEP 07 (2009) 040 [arXiv:0905.2559] [INSPIRE].

[89] T. Hambye and K. Riesselmann, Matching conditions and Higgs mass upper bounds revisited, Phys. Rev. D 55 (1997) 7255 [hep-ph/9610272] [INSPIRE].

[90] K. Riesselmann and S. Willenbrock, Ruling out a strongly interacting standard Higgs model, Phys. Rev. D 55 (1997) 311 [hep-ph/9608280] [INSPIRE].

[91] M.E. Peskin and T. Takeuchi, A New constraint on a strongly interacting Higgs sector, Phys. Rev. Lett. 65 (1990) 964 [INSPIRE].

[92] M.E. Peskin and T. Takeuchi, Estimation of oblique electroweak corrections, Phys. Rev. D 46 (1992) 381 [INSPIRE].

[93] W. Grimus, L. Lavoura, O.M. Ogreid and P. Osland, The Oblique parameters in multi-Higgs-doublet models, Nucl. Phys. B 801 (2008) 81 [arXiv:0802.4353] [InSPIRE].

[94] S. Profumo, M.J. Ramsey-Musolf, C.L. Wainwright and P. Winslow, Singlet-catalyzed electroweak phase transitions and precision Higgs boson studies, Phys. Rev. D 91 (2015) 035018 [arXiv: 1407.5342] [INSPIRE].

[95] J. Haller, A. Hoecker, R. Kogler, K. Mönig, T. Peiffer and J. Stelzer, Update of the global electroweak fit and constraints on two-Higgs-doublet models, Eur. Phys. J. C 78 (2018) 675 [arXiv: 1803.01853] [INSPIRE]. 
[96] P. Bechtle et al., HiggsBounds - 4: Improved Tests of Extended Higgs Sectors against Exclusion Bounds from LEP, the Tevatron and the LHC, Eur. Phys. J. C 74 (2014) 2693 [arXiv: 1311.0055] [INSPIRE].

[97] P. Bechtle, S. Heinemeyer, O. Stål, T. Stefaniak and G. Weiglein, HiggsSignals: Confronting arbitrary Higgs sectors with measurements at the Tevatron and the LHC, Eur. Phys. J. C 74 (2014) 2711 [arXiv:1305.1933] [InSPIRE].

[98] ATLAS collaboration, Measurements of the Higgs boson production and decay rates and coupling strengths using pp collision data at $\sqrt{s}=7$ and $8 \mathrm{TeV}$ in the ATLAS experiment, Eur. Phys. J. C 76 (2016) 6 [arXiv:1507.04548] [inSPIRE].

[99] CMS collaboration, Observation of the Diphoton Decay of the Higgs Boson and Measurement of Its Properties, Eur. Phys. J. C 74 (2014) 3076 [arXiv:1407.0558] [INSPIRE].

[100] CMS collaboration, Measurement of Higgs Boson Production and Properties in the $W W$ Decay Channel with Leptonic Final States, JHEP 01 (2014) 096 [arXiv:1312.1129] [INSPIRE].

[101] CMS collaboration, Measurement of the Properties of a Higgs Boson in the Four-Lepton Final State, Phys. Rev. D 89 (2014) 092007 [arXiv: 1312.5353] [INSPIRE].

[102] CMS collaboration, Evidence for the direct decay of the $125 \mathrm{GeV}$ Higgs boson to fermions, Nature Phys. 10 (2014) 557 [arXiv:1401.6527] [INSPIRE].

[103] D. Barducci et al., Collider limits on new physics within MicrOMEGAs_4.3, Comput. Phys. Commun. 222 (2018) 327 [arXiv:1606.03834] [INSPIRE].

[104] Planck collaboration, Planck 2015 results. XIII. Cosmological parameters, Astron. Astrophys. 594 (2016) A13 [arXiv:1502.01589] [INSPIRE].

[105] R. Apreda, M. Maggiore, A. Nicolis and A. Riotto, Gravitational waves from electroweak phase transitions, Nucl. Phys. B 631 (2002) 342 [gr-qc/0107033] [InSPIRE].

[106] C.L. Wainwright, CosmoTransitions: Computing Cosmological Phase Transition Temperatures and Bubble Profiles with Multiple Fields, Comput. Phys. Commun. 183 (2012) 2006 [arXiv: 1109.4189] [INSPIRE].

[107] M. Kamionkowski, A. Kosowsky and M.S. Turner, Gravitational radiation from first order phase transitions, Phys. Rev. D 49 (1994) 2837 [astro-ph/9310044] [INSPIRE].

[108] J.R. Espinosa, T. Konstandin, J.M. No and G. Servant, Energy Budget of Cosmological First-order Phase Transitions, JCAP 06 (2010) 028 [arXiv:1004.4187] [INSPIRE].

[109] J.M. No, Large Gravitational Wave Background Signals in Electroweak Baryogenesis Scenarios, Phys. Rev. D 84 (2011) 124025 [arXiv:1103.2159] [INSPIRE].

[110] J. Ellis, M. Lewicki and J.M. No, On the Maximal Strength of a First-Order Electroweak Phase Transition and its Gravitational Wave Signal, JCAP 04 (2019) 003 [arXiv: 1809.08242] [INSPIRE].

[111] C. Caprini et al., Science with the space-based interferometer eLISA. II: Gravitational waves from cosmological phase transitions, JCAP 04 (2016) 001 [arXiv: 1512.06239] [INSPIRE].

[112] R.-G. Cai, M. Sasaki and S.-J. Wang, The gravitational waves from the first-order phase transition with a dimension-six operator, JCAP 08 (2017) 004 [arXiv: 1707.03001] [INSPIRE]. 
[113] S.J. Huber and T. Konstandin, Gravitational Wave Production by Collisions: More Bubbles, JCAP 09 (2008) 022 [arXiv:0806.1828] [INSPIRE].

[114] R. Jinno and M. Takimoto, Gravitational waves from bubble collisions: An analytic derivation, Phys. Rev. D 95 (2017) 024009 [arXiv:1605.01403] [INSPIRE].

[115] M. Hindmarsh, S.J. Huber, K. Rummukainen and D.J. Weir, Numerical simulations of acoustically generated gravitational waves at a first order phase transition, Phys. Rev. D 92 (2015) 123009 [arXiv: 1504.03291] [INSPIRE].

[116] C. Caprini, R. Durrer and G. Servant, The stochastic gravitational wave background from turbulence and magnetic fields generated by a first-order phase transition, JCAP 12 (2009) 024 [arXiv:0909.0622] [INSPIRE].

[117] D. Bödeker and G.D. Moore, Electroweak Bubble Wall Speed Limit, JCAP 05 (2017) 025 [arXiv:1703.08215] [INSPIRE].

[118] N. Seto, S. Kawamura and T. Nakamura, Possibility of direct measurement of the acceleration of the universe using $0.1-\mathrm{Hz}$ band laser interferometer gravitational wave antenna in space, Phys. Rev. Lett. 87 (2001) 221103 [astro-ph/0108011] [INSPIRE].

[119] T. Robson, N.J. Cornish and C. Liu, The construction and use of LISA sensitivity curves, Class. Quant. Grav. 36 (2019) 105011 [arXiv: 1803.01944] [INSPIRE].

[120] S. Isoyama, H. Nakano and T. Nakamura, Multiband Gravitational-Wave Astronomy: Observing binary inspirals with a decihertz detector, B-DECIGO, PTEP 2018 (2018) 073E01 [arXiv: 1802.06977] [INSPIRE].

[121] K. Yagi, N. Tanahashi and T. Tanaka, Probing the size of extra dimension with gravitational wave astronomy, Phys. Rev. D 83 (2011) 084036 [arXiv:1101.4997] [INSPIRE].

[122] K. Yagi, Scientific Potential of DECIGO Pathfinder and Testing GR with Space-Borne Gravitational Wave Interferometers, Int. J. Mod. Phys. D 22 (2013) 1341013 [arXiv: 1302.2388] [INSPIRE].

[123] K. Farakos, K. Kajantie, K. Rummukainen and M.E. Shaposhnikov, $3-D$ physics and the electroweak phase transition: Perturbation theory, Nucl. Phys. B 425 (1994) 67 [hep-ph/9404201] [INSPIRE].

[124] E. Braaten and A. Nieto, Effective field theory approach to high temperature thermodynamics, Phys. Rev. D 51 (1995) 6990 [hep-ph/9501375] [INSPIRE].

[125] K. Kajantie, M. Laine, K. Rummukainen and M.E. Shaposhnikov, Generic rules for high temperature dimensional reduction and their application to the standard model, Nucl. Phys. B 458 (1996) 90 [hep-ph/9508379] [INSPIRE].

[126] T.s. Brauner, T.V.I. Tenkanen, A. Tranberg, A. Vuorinen and D.J. Weir, Dimensional reduction of the Standard Model coupled to a new singlet scalar field, JHEP 03 (2017) 007 [arXiv: 1609.06230] [INSPIRE].

[127] O. Gould, J. Kozaczuk, L. Niemi, M.J. Ramsey-Musolf, T.V.I. Tenkanen and D.J. Weir, Nonperturbative analysis of the gravitational waves from a first-order electroweak phase transition, Phys. Rev. D 100 (2019) 115024 [arXiv:1903.11604] [INSPIRE].

[128] F. Staub, Exploring new models in all detail with SARAH, Adv. High Energy Phys. 2015 (2015) 840780 [arXiv: 1503.04200] [inSPIRE]. 